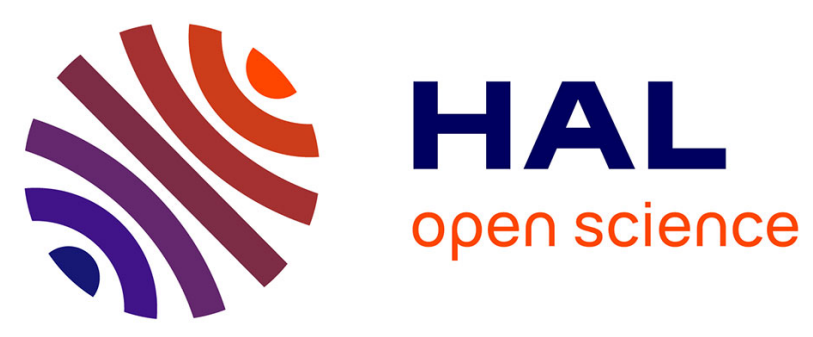

\title{
An Algorithm to Compute the Inverse Image of a Point with Respect to a Nondeterministic Max Plus Linear System
}

Renato Markele Ferreira Cândido, Laurent Hardouin, Mehdi Lhommeau, Rafael Santos-Mendes

\section{To cite this version:}

Renato Markele Ferreira Cândido, Laurent Hardouin, Mehdi Lhommeau, Rafael Santos-Mendes. An Algorithm to Compute the Inverse Image of a Point with Respect to a Nondeterministic Max Plus Linear System. IEEE Transactions on Automatic Control, 2020, Volume: 66, Issue: 4, April 2021, pp.1-1. 10.1109/TAC.2020.2998726 . hal-02961770

\section{HAL Id: hal-02961770 \\ https://univ-angers.hal.science/hal-02961770}

Submitted on 8 Oct 2020

HAL is a multi-disciplinary open access archive for the deposit and dissemination of scientific research documents, whether they are published or not. The documents may come from teaching and research institutions in France or abroad, or from public or private research centers.
L'archive ouverte pluridisciplinaire HAL, est destinée au dépôt et à la diffusion de documents scientifiques de niveau recherche, publiés ou non, émanant des établissements d'enseignement et de recherche français ou étrangers, des laboratoires publics ou privés. 


\title{
An Algorithm to Compute the Inverse Image of a Point with Respect to a Nondeterministic Max Plus Linear System
}

\author{
Renato Markele Ferreira Cândido, Laurent Hardouin, Mehdi Lhommeau and Rafael Santos Mendes
}

\begin{abstract}
Max Plus Linear (MPL) systems are often described by a transition function, which models the state evolution of the system, and a measurement function, which binds the measures with the system states. Methods for computing the inverse image of a point w.r.t. the measurement function are particularly interesting in applications where it is desirable to obtain informations about the system states based on the output observations. The inverse image of a set w.r.t. a nondeterministic MPL system, called uncertain MPL (uMPL) system, can be computed by using the Difference-Bound Matrices (DBM) approach. In this work we aim to use an interval analysis to propose a method to compute the inverse image of a point w.r.t. an uMPL system. The algorithm proposed has a lower worst-case complexity compared with the DBM approach as previously proposed in the literature.
\end{abstract}

Index Terms-Max Plus Linear Systems, Nondeterministic Systems, Inverse Image, Interval Analysis.

\section{INTRODUCTION}

D ISCRETE Event Dynamic Systems (DEDS) are discretestate systems whose dynamics are entirely driven by the occurrence of asynchronous events over discrete time instants [1, Sec. 1.3.2]. DEDS subjected only to synchronization and time delay phenomena can be described in terms of linear equations using the Max Plus Algebra. The Max Plus Algebra is an idempotent semiring, an algebraic structure also called dioid [2], in which the operations of sum $(\oplus)$ and product $(\otimes)$ are defined as the maximization and addition, respectively. Synchronization phenomena are modeled thanks to maximization: the start of a task waits for the completion of the preceding tasks, while the delay phenomena are modeled thanks to the classical sum: the completion time of a task is equal to the starting time plus the task duration. The Max Plus Linear (MPL) equations are used to model manufacturing systems, telecommunication networks, railway networks, and parallel computing [2], [3]. The linearity property has advantaged the emergence of a specific theory for the performance analysis [4] and the control of these systems, e.g., optimal open loop control [5], [6] and optimal state-feedback control. Among closed-loop strategies we can cite the model matching problem [7] and the control strategies allowing the state to stay in a specific state subspace or semimodule [8], [9], [10], [11], [12].

R. Cândido and R. Mendes are with the Department of Computer Engineering and Industrial Automation - DCA, School of Electrical and Computer Engineering, University of Campinas - UNICAMP - 13083-970 Campinas, SP, Brazil.

L. Hardouin and M. Lhommeau are with Laboratoire Angevin de Recherche en Ingénierie des Systèmes - Polytech Angers - Université d'Angers, 62 av. Notre Dame du Lac, 49100 Angers, France.
In order to control and analyze a dynamic system, the knowledge of the system states is required. For this reason, the development of methods for observing and estimating the system states are of great interest. In [13] a dynamic observer for MPL systems is proposed and in [14] a Luenberger inspired observer [15] is developed. In both cases, uncertainties w.r.t. the model parameters are considered, however, the probabilistic aspects are not taken into account. Though, these probabilistic aspects are of great interest in the filtering problems where the model parameters are influenced by random processes. The filtering problem consists in estimating the state of a stochastic system. The Stochastic Max Plus Linear (SMPL) systems are defined as MPL systems where the matrices entries are characterized by random variables [16], [17], [18], [19], [20]. In this work, although the probabilistic aspects of the uncertainties are not considered, we are interested in systems where the uncertain parameters can vary over a known interval. Formally, we define the uncertain Max Plus Linear (uMPL) systems as nondeterministic MPL systems where, at each event step, the entries of the system matrices can take an arbitrary value within a real interval. In [21], [22], [23] the filtering problem for uMPL systems is addressed by considering the Sequential Monte-Carlo (SMC) method, also known as Particle Filters.

The design of filters can take advantage of the conditional reachability analysis. The reachability analysis aims to compute the set of all states that can be reached from a set of initial conditions. In [24] reachability analysis of timed automata is tackled by considering max-plus polyhedra ${ }^{1}$. An important drawback in this approach is that the analysis of timed automata often requires to express strict constraints while max-plus polyhedra are by definition topologically closed. A generalization of max-plus polyhedra is introduced in [26], where the authors define a semiring of germs which allows to handle a class of non-necessarily closed max-plus polyhedra. In [27], the reachability analysis of MPL systems is tackled by considering the Difference-Bound Matrices (DBM). In [28] the approach is extended to uMPL systems and the results are used to solve the conditional reachability problem, which is defined as the computation of the set of all states that may be reached from a set of initial states, in a given number of event steps, conditioned to a sequence of measures. The solution of this problem involves the computation of the image of sets and the inverse image of points. The both can be done by using the

${ }^{1}$ For a more exhaustive presentation on max-plus polyhedra, see [25] 
DBM approach. However, as it will be shown in this paper, the inverse image of a point can be computed by considering a more efficient approach from a computational point of view. Indeed, we propose an alternative procedure, based on interval analysis, to compute the inverse image of a point w.r.t. an uMPL system. It will be shown that the inverse image of a point can be represented by a collection of hyperrectangles. A hyperrectangle can be completely characterized by an interval matrix. Although the DBM can represent a more general class of sets, including hyperrectangle, the interval matrix yields a simpler and less expensive representation for the inverse image of a point. For this reason, the proposed procedure has worstcase complexity lower than the DBM approach. Therefore, the procedure presented in this work can be used to solve the conditional reachability problem, and more precisely, to reduce the computational effort to get the inverse image of the measures, which is a key point of this problem. It must be noted that, the DBM approach will still be required to compute the direct image of the sets.

Both DBM and Hyperrectangles approaches have exponential complexity, which limits the dimension of the addressable problem. On the other hand, the complexity of the algorithms involving max-plus polyhedra are in general polynomial. In this sense, approaches based on max-plus polyhedra seems to be a promising way to reduce the complexity of reachability computations for MPL systems. However, to the best of the authors' knowledge, there are no approaches based on maxplus polyhedra for solving the forward and the backward reachability problem for general MPL systems and such methods remain to be designed.

Computing the inverse image of a point w.r.t an uMPL equation consists in computing the solutions of MPL equations where the coefficients belong to intervals, see e.g. [29]. In [30], necessary and sufficient conditions for weakly and strongly solvability of interval systems of max-separable linear equations are provided. In [31] the tolerance solvable, the weakly tolerance solvable and the strongly tolerance solvable systems are introduced. The present work provides a method to compute the complete set of solutions of uMPL equations, a special class of interval systems of max-separable linear equations.

The technique discussed in this work is implemented in MATLAB and the codes are freely available for download at [32].

The paper is organized as follows: Section II recalls the algebraic tools used in the next sections. Section III presents the main contribution: an approach to compute the inverse image of a point w.r.t. an uMPL system based on the interval analysis. Section IV yields an application of the results in a filtering problem of an uMPL system. Finally, Section V concludes the work.

\section{PReliminaries}

\section{A. Interval Analysis}

Interval arithmetic is presented in [33] and extended to Max Plus Algebra in [34], [35], [6], [36]. An interval can be defined by a pair of entries of the type $(a, \bowtie)$ as follows:

$$
[x]=[(\underline{x}, \underline{\bowtie}),(\bar{x}, \bar{\bowtie})]=\{x \in \mathbb{R}: \underline{x} \underline{\bowtie} x \bar{\bowtie} \bar{x}\} .
$$

where $\underline{x} \in \mathbb{R} \cup\{-\infty\}$ and $\bar{x} \in \mathbb{R} \cup\{+\infty\}$ are, respectively, the lower and upper bounds of the interval. The signs $\bowtie \in\{\geq,>\}$ and $\bar{\bowtie} \in\{<, \leq\}$ define whether the interval is closed (if $\bowtie=\geq$ and $\bar{\bowtie}=\leq$ ), open (if $\bowtie=>$ and $\bar{\bowtie}=<$ ) or halfclosed/half-open (otherwise).

In order to define the intersection of two intervals, a partial order must be defined. Consider two typical interval entries $(a, \bowtie)$ and $\left(a^{\prime}, \bowtie^{\prime}\right)$. The pairs $(a, \bowtie)$ and $\left(a^{\prime}, \bowtie^{\prime}\right)$ are ordered if $\bowtie, \bowtie^{\prime} \in\{\geq,>\}$ or if $\bowtie, \bowtie^{\prime} \in\{<, \leq\}$. In this case, $(a, \bowtie) \preceq\left(a^{\prime}, \bowtie^{\prime}\right)$ if $\left(a<a^{\prime}\right)$ or $^{2}\left(a=a^{\prime}\right.$ and $\left.\bowtie \leq \bowtie^{\prime}\right)$. On the other hand, the pairs $(a, \bowtie)$ and $\left(a^{\prime}, \bowtie^{\prime}\right)$ are not comparable if $\bowtie \in\{\geq,>\}$ and $\bowtie^{\prime} \in\{<, \leq\}$ or if $\bowtie \in\{<, \leq\}$ and $\bowtie^{\prime} \in\{\geq,>\}$.

Given this partial order, the intersection of $[x]=$ $\left[\left(\underline{x}, \underline{\bowtie}_{x}\right),\left(\bar{x}, \bar{\bowtie}_{x}\right)\right]$ and $[y]=\left[\left(\underline{y}, \underline{凶}_{y}\right),\left(\bar{y}, \bar{\bowtie}_{y}\right)\right]$ can be defined as:

$[x] \cap[y]=\left[\max \left\{\left(\underline{x}, \underline{\bowtie}_{x}\right),\left(\underline{y}, \underline{凶}_{y}\right)\right\}, \min \left\{\left(\bar{x}, \bar{\bowtie}_{x}\right),\left(\bar{y}, \bar{\bowtie}_{y}\right)\right\}\right]$.

Remark 1: An interval $[x]$ is empty if $\underline{x}>\bar{x}$; or if $\underline{x}=\bar{x}$ and $\bowtie=>$; or if $\underline{x}=\bar{x}$ and $\bar{\bowtie}=<$. For instance, the following intervals are empty: $[(5, \geq),(3, \leq)]$, $[(5,>),(5, \leq)],[(5, \geq),(5,<)]$.

In general, the union of intervals cannot be represented as a single interval, but by collection of intervals. However, if the intervals have nonempty intersection, the union is an interval defined by:

$$
[x] \cup[y]=\left[\min \left\{\left(\underline{x}, \underline{凶}_{x}\right),\left(\underline{y}, \underline{凶}_{y}\right)\right\}, \quad \max \left\{\left(\bar{x}, \bar{\bowtie}_{x}\right),\left(\bar{y}, \bar{\bowtie}_{y}\right)\right\}\right] .
$$

Example 1: Consider the intervals:

$$
\begin{aligned}
& {[x]=[(0,>),(5,<)]=\{x \in \mathbb{R}: 0<x<5\}} \\
& {[y]=[(3,>),(6, \leq)]=\{x \in \mathbb{R}: 3<x \leq 6\}} \\
& {[z]=[(7, \geq),(10, \leq)]=\{x \in \mathbb{R}: 7 \leq x \leq 10\}}
\end{aligned}
$$

According to (2) and (3):

$$
\begin{aligned}
& {[x] \cap[y]=[(3,>),(5,<)]=\{x \in \mathbb{R}: 3<x<5\}} \\
& {[x] \cup[y]=[(0,>),(6, \leq)]=\{x \in \mathbb{R}: 0<x \leq 6\}}
\end{aligned}
$$

Note that $[x] \cap[z]=\emptyset=[y] \cap[z]$. In this case, equation (3) cannot be used to compute neither $[x] \cup[z]$ nor $[y] \cup[z]$. Indeed, $[x] \cup[z] \equiv\{x \in \mathbb{R}: 0<x<5\} \cup\{x \in \mathbb{R}: 7 \leq x \leq 10\}$, which cannot be represented by a single interval. The same holds for $[y] \cup[z]$.

An interval matrix is herein defined as a matrix whose entries are intervals, they will be noted by a bold capital letter in brackets. The intersection of two interval matrices can be computed as the element-wise intersection of the corresponding entries. Thus, given a set of $M$ interval matrices $\left[\mathbf{A}_{\mathbf{1}}\right], \ldots,\left[\mathbf{A}_{\mathbf{M}}\right]$ of dimension $n \times p$, the intersection $\left[\mathbf{A}_{\mathbf{1}}\right] \cap \cdots \cap\left[\mathbf{A}_{\mathbf{M}}\right]$ is defined by:

$$
\left(\bigcap_{r=1}^{M}\left[\mathbf{A}_{\mathbf{r}}\right]\right)_{i j}=\bigcap_{r=1}^{M}\left[a_{r_{i j}}\right]
$$

${ }^{2}$ The symbols $<, \leq, \geq$ and $>$ are assumed to be partially ordered with $<$ strictly less than $\leq$ and $\geq$ strictly less than $>$. 
where $\left[a_{r_{i j}}\right]$ is the entry in the $i$-th row and $j$-column of matrix $\left[\mathbf{A}_{\mathbf{r}}\right]$. It should be remarked that, in general, the union of interval matrices in $\mathbb{R}^{n \times p}$ cannot be represented by a single interval matrix but by a collection of interval matrices.

A hyperrectangle in $\mathbb{R}^{n}$, defined as the set $\left\{\mathbf{x} \in \mathbb{R}^{n}\right.$ : $\left.\underline{x}_{1} \underline{凶}_{1} x_{1} \bar{凶}_{1} \bar{x}_{1}, \ldots, \underline{x}_{n} \underline{凶}_{n} x_{n} \bar{\bowtie}_{n} \bar{x}_{n}\right\}$, can be represented by an $n \times 1$ interval matrix:

$$
[\mathbf{x}]=\left(\begin{array}{c}
\left.\left[\underline{x}_{1}, \underline{\bowtie}_{1}\right),\left(\bar{x}_{1}, \bar{\bowtie}_{1}\right)\right] \\
\vdots \\
\left.\left(\underline{x}_{n}, \underline{凶}_{n}\right),\left(\bar{x}_{n}, \bar{\bowtie}_{n}\right)\right]
\end{array}\right) .
$$

Remark 2: Any vector $\mathbf{x} \in \mathbb{R}^{n}$ can be represented by a matrix of degenerate intervals, in which $\underline{x}_{i}=\bar{x}_{i}=x_{i}$ and ${\unrhd_{i}}_{i}=\geq, \bar{\bowtie}_{i}=\leq$.

\section{B. Max Plus Linear Systems}

A set $S$, endowed with two internal operations: sum $(\oplus)$ and product $(\otimes)$ is a dioid or idempotent semiring if the sum is associative, commutative and idempotent (i.e. $a \oplus a=a$ ) and the product is associative and left and right distributive ${ }^{3}$ w.r.t. the sum. The null (or zero) element, denoted by $\varepsilon$, is such that $\forall a \in S, a \oplus \varepsilon=a$, and the identity element, denoted by $e$, is such that $\forall a \in S, a \otimes e=e \otimes a=a$. Besides, the zero element is absorbing for the $\otimes$ operation (i.e. $\forall a \in S, a \otimes \varepsilon=$ $\varepsilon \otimes a=\varepsilon$ ) [2, Def. 4.1]. In this algebraic structure, a partial order relation is defined by:

$$
a \succeq b \Leftrightarrow a=a \oplus b .
$$

Given these conditions, it appears that the set $\overline{\mathbb{R}}_{\max }:=\mathbb{R} \cup$ $\{-\infty\} \cup\{\infty\}$ and the operations: $\alpha \oplus \beta \equiv \max \{\alpha, \beta\}$ and $\alpha \otimes$ $\beta \equiv \alpha+\beta$, with $\varepsilon=-\infty, e=0$, and with the convention that $\infty \otimes \varepsilon=\varepsilon$, is a dioid [2, Def. 3.4]. Moreover, it can be stated that this is a complete dioid since it is closed for infinite sums and the left and right distributivity of the product extend to infinite sums. This structure is called the completed Max Plus semiring.

For complete dioids, the order relation (6) can be written as: $a \succeq b \Leftrightarrow a=a \oplus b \Leftrightarrow b=a \wedge b$, where $a \wedge b$ is the greatest lower bound of $a$ and $b$. The Max Plus operations can be extended to matrices as follows. If $\mathbf{A}, \mathbf{B} \in \overline{\mathbb{R}}_{\max }^{n \times p}$ and $\mathbf{C} \in \overline{\mathbb{R}}_{\max }^{p \times q}$, then:

$$
\begin{aligned}
& {[\mathbf{A} \oplus \mathbf{B}]_{i j}=a_{i j} \oplus b_{i j}, \quad[\mathbf{A} \otimes \mathbf{C}]_{i j}=\bigoplus_{k=1}^{p} a_{i k} \otimes c_{k j},} \\
& {[\mathbf{A} \wedge \mathbf{B}]_{i j}=a_{i j} \wedge b_{i j} .}
\end{aligned}
$$

Moreover, the Max Plus operations can be extended to intervals as follows. Consider two ordered interval entries $(a, \bowtie)$ and $\left(a^{\prime}, \bowtie^{\prime}\right)$, then:

$$
(a, \bowtie) \oplus\left(a^{\prime}, \bowtie^{\prime}\right)= \begin{cases}(a, \bowtie) & \text { if }(a, \bowtie) \succeq\left(a^{\prime}, \bowtie^{\prime}\right), \\ \left(a^{\prime}, \bowtie^{\prime}\right) & \text { otherwise. }\end{cases}
$$

$(a, \bowtie) \otimes\left(a^{\prime}, \bowtie^{\prime}\right)= \begin{cases}\left(a \otimes a^{\prime}, \min \left(\bowtie, \bowtie^{\prime}\right)\right) & \text { if } \bowtie, \bowtie^{\prime} \in\{<, \leq\}, \\ \left(a \otimes a^{\prime}, \max \left(\bowtie, \bowtie^{\prime}\right)\right) & \text { if } \bowtie, \bowtie^{\prime} \in\{\geq,>\} .\end{cases}$

\footnotetext{
${ }^{3}$ The product is not necessarily commutative.
}

Hence:

$$
\begin{aligned}
& {[x] \oplus[y]=\left[\left(\underline{x}, \unrhd_{x}\right) \oplus\left(\underline{y}, \unrhd_{y}\right),\left(\bar{x}, \bar{\bowtie}_{x}\right) \oplus\left(\bar{y}, \bar{\bowtie}_{y}\right)\right](10)} \\
& {[x] \otimes[y]=\left[\left(\underline{x}, \unrhd_{x}\right) \otimes\left(\underline{y}, \underline{凶}_{y}\right),\left(\bar{x}, \bar{\bowtie}_{x}\right) \otimes\left(\bar{y}, \bar{\bowtie}_{y}\right)\right](11)}
\end{aligned}
$$

Recalling Remark 2, given a point $d \in \mathbb{R}$, we have that:

$$
\begin{aligned}
& {[x] \oplus d=\left[\left(\underline{x}, \unrhd_{x}\right) \oplus(d, \geq),\left(\bar{x}, \bar{\rtimes}_{x}\right) \oplus(d, \leq)\right],} \\
& {[x] \otimes d=\left[\left(\underline{x} \otimes d, \underline{凶}_{x}\right),\left(\bar{x} \otimes d, \bar{\bowtie}_{x}\right)\right] .}
\end{aligned}
$$

The $\oplus$ and $\otimes$ are extended to interval matrices as follows: If $[\mathbf{A}],[\mathbf{B}]$ and $[\mathbf{C}]$ are, respectively, $n \times p, n \times p$ and $p \times q$ interval matrices, then:

$$
([\mathbf{A}] \oplus[\mathbf{B}])_{i j}=\left[a_{i j}\right] \oplus\left[b_{i j}\right], \quad([\mathbf{A}] \otimes[\mathbf{C}])_{i j}=\bigoplus_{k=1}^{p}\left(\left[a_{i k}\right] \otimes\left[c_{k j}\right]\right) .
$$

In the following, the MPL systems are introduced. The autonomous model of an MPL system is given by:

$$
\mathbf{x}(k)=\mathbf{A} \otimes \mathbf{x}(k-1),
$$

where the entries of matrix $\mathbf{A} \in \overline{\mathbb{R}}_{\text {max }}^{n \times n}$ are the parameters of the model. The variable $k \in \mathbb{N}$ is an event-number and the state vector $\mathbf{x} \in \overline{\mathbb{R}}_{\text {max }}^{n}$ is a dater, i.e, $\mathbf{x}(k)$ contains the $k$-th date of occurrence of each event of the system.

The nonautonomous model of an MPL system is defined as:

$$
\mathbf{x}(k)=\mathbf{A} \otimes \mathbf{x}(k-1) \oplus \mathbf{B} \otimes \mathbf{u}(k),
$$

where $\mathbf{u}$ is an external input vector and $\mathbf{B} \in \overline{\mathbb{R}}_{\max }^{n \times m}$.

Any nonautonomous MPL system can be transformed into an augmented autonomous MPL model by considering $\mathbf{M}=$ $(\mathbf{A ~ B}) \in \overline{\mathbb{R}}_{\max }^{n \times(n+m)}$ and $\mathbf{y}(k-1)=\left(\mathbf{x}(k-1)^{T} \mathbf{u}(k)^{T}\right)^{T}[2$, Sec. 2.5.4].

$$
\mathbf{x}(k)=\mathbf{M} \otimes \mathbf{y}(k-1) .
$$

To model uncertain systems, the entries of matrix $\mathbf{A}$ are assumed to be nondeterministic. This is consistent with the assumption that the entries of $\mathbf{A}$ are associated to the system delays, that are subject to variations due to disturbances. Formally, it is assumed that at each event step $k$ the system matrix entries can take an arbitrary value within a real interval. The autonomous model of an uncertain MPL (uMPL) system is given by:

$$
\mathbf{x}(k)=\mathbf{A}(k) \otimes \mathbf{x}(k-1), \mathbf{A}(k) \in[\mathbf{A}], \mathbf{A}(k) \in \overline{\mathbb{R}}_{\text {max }}^{n \times n},
$$

where, at each event step $k$, the entries of $\mathbf{A}(k)$ can take an arbitrary value in closed real intervals. Hence, $a_{i j}(k) \in$ $\left[\left(\underline{a}_{i j}, \geq\right),\left(\bar{a}_{i j}, \leq\right)\right]$. The system is assumed to be such that $\mathbf{x}(k) \succeq \mathbf{x}(k-1)$ and it works according to FIFO (first in, first out) rule. Hence, matrix $\mathbf{A}(k)$ is assumed to be such that $a_{i i}(k) \succeq e$, i.e, $\mathbf{A}(k) \succeq I_{n}$, the identity matrix in $\overline{\mathbb{R}}_{\text {max }}^{n \times n}$.

Notation 1: Recall that the entries of an UMPL system matrix are assumed to be in closed real intervals, i.e., the entries of $[\mathbf{A}]$ are of the type $\left[a_{i j}\right]=[(\underline{a}, \geq),(\bar{a}, \leq)]$, where $\underline{a}, \bar{a} \in \overline{\mathbb{R}}_{\max }$. The signs $\geq$ and $\leq$ can be omitted and these entries can be represented as simply $\left[a_{i j}\right]=[\underline{a}, \bar{a}]$. This simplified representation of closed intervals will be considered to express interval matrices that represent uMPL systems. 
Hyperrenctangles will still be represented by considering the notation presented in section II-A.

Notation 2: Given an interval matrix $[\mathbf{A}]$ representing an uMPL system, we denote by $\underline{\mathbf{A}}$ and $\overline{\mathbf{A}}$ the matrices of lower and upper bounds of $[\mathbf{A}]$, respectively. Hence, $\underline{\mathbf{A}}$ has entries $\underline{a}_{i j}$ and $\overline{\mathbf{A}}$ has entries $\bar{a}_{i j}$.

Example 2: Consider the following uMPL system: $\mathbf{x}(k)=$ $\mathbf{A}(k) \otimes \mathbf{x}(k-1), \quad \mathbf{A}(k) \in[\mathbf{A}]$, where

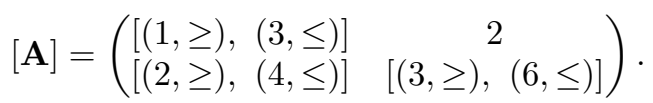

By considering Notations 1 and 2, the interval matrix $[\mathbf{A}]$ and the matrices of lower and upper bounds can be represented, respectively as:

$$
[\mathbf{A}]=\left(\begin{array}{cc}
{[1,3]} & 2 \\
{[2,4} & {[3,6}
\end{array}\right), \quad \underline{\mathbf{A}}=\left(\begin{array}{ll}
1 & 2 \\
2 & 3
\end{array}\right), \quad \overline{\mathbf{A}}=\left(\begin{array}{ll}
3 & 2 \\
4 & 6
\end{array}\right) .
$$

Similarly to MPL systems, any nonautonomous uMPL system can be transformed into an augmented autonomous uMPL model, see (17).

\section{Residuation}

Residuation theory [2] deals with solution of equation $f(x) \preceq y$ in semiring. In particular, in $\overline{\mathbb{R}}_{\max }$, it yields the way to compute the greatest solution of $\mathbf{A} \otimes \mathbf{X} \preceq \mathbf{B}$, with $\mathbf{A} \in \overline{\mathbb{R}}_{\max }^{n \times p}, \mathbf{X} \in \overline{\mathbb{R}}_{\max }^{p \times m}$ and $\mathbf{B} \in \overline{\mathbb{R}}_{\max }^{n \times m}$. This greatest solution is denoted $\hat{\mathbf{X}}=\mathbf{A} \phi \mathbf{B}$ and achieves equality if it exists. Each entry of $\hat{\mathbf{X}}$ is computed as follows:

$$
\hat{x}_{i j}=\bigwedge_{k=1}^{n}\left(a_{k i} \phi b_{k j}\right)
$$

where $a_{k i} \phi b_{k j}=b_{k j}-a_{k i}$ and then it is the greatest solution of equality $a_{k i} \otimes x=b_{k j}$. In the same way, the greatest solution of inequality $\mathbf{X} \otimes \mathbf{C} \preceq \mathbf{B}$, with $\mathbf{X} \in \overline{\mathbb{R}}_{\max }^{n \times p}, \mathbf{C} \in \overline{\mathbb{R}}_{\max }^{p \times m}$ and $\mathbf{B} \in \overline{\mathbb{R}}_{\max }^{n \times m}$, is denoted $\hat{\mathbf{X}}=\mathbf{B} \phi \mathbf{C}$ and is computed by

$$
\hat{x}_{i j}=\bigwedge_{k=1}^{m}\left(b_{i k} \phi c_{j k}\right)=\bigwedge_{k=1}^{m}\left(b_{i k}-c_{j k}\right) .
$$

From these definitions it is useful to remark that the system $\mathbf{A} \otimes \mathbf{x} \preceq \mathbf{b}$, with $\mathbf{A} \in \overline{\mathbb{R}}_{\max }^{n \times p}, \mathbf{x} \in \overline{\mathbb{R}}_{\max }^{p \times 1}$ and $\mathbf{b} \in \overline{\mathbb{R}}_{\max }^{n \times 1}$ yields the greatest $\hat{\mathbf{x}}=\mathbf{A} \phi \mathbf{b}$ with

$$
\hat{x}_{j}=\bigwedge_{k=1}^{n}\left(a_{k j} \phi b_{k}\right)=\bigwedge_{k=1}^{n}\left(b_{k}-a_{k j}\right)
$$

and the greatest $\mathbf{A}$ is given by $\hat{\mathbf{A}}=\mathbf{b} \phi \mathbf{x}$ with

$$
\hat{a}_{i j}=b_{i} \phi x_{j}=b_{i}-x_{j} .
$$

Hence, this $\hat{\mathbf{A}}$ achieves equality, i.e, $\hat{\mathbf{A}} \otimes \mathbf{x}=\mathbf{b}$. Furthermore, it should be remarked that the greatest solution of the system $\mathbf{A} \otimes \mathbf{x} \preceq b$, with $\mathbf{A} \in \overline{\mathbb{R}}_{\text {max }}^{1 \times p}, \mathbf{x} \in \overline{\mathbb{R}}_{\text {max }}^{p \times 1}$ and $b \in \overline{\mathbb{R}}_{\text {max }}$ is given by $\hat{\mathbf{x}}=\mathbf{A} \phi b$ with

$$
\hat{x}_{j}=a_{1 j} \phi b=b-a_{1 j} .
$$

Hence,

$$
\mathbf{A} \otimes \hat{\mathbf{x}}=\bigoplus_{j=1}^{p} a_{1 j} \otimes \hat{x}_{j}=\bigoplus_{j=1}^{p} a_{1 j} \otimes\left(b-a_{1 j}\right)=b,
$$

i.e., $\hat{\mathbf{x}}$ is the greatest vector ensuring $\mathbf{A} \otimes \hat{\mathbf{x}}=b$.

\section{Image AND InVERse Image OF A POINT}

This section presents an approach to compute the image and the inverse image of a point w.r.t. the uMPL equation described by:

$$
\mathbf{z}=\mathbf{A} \otimes \mathbf{x}, \mathbf{A} \in[\mathbf{A}], \mathbf{A} \in \overline{\mathbb{R}}_{\max }^{n \times p},
$$

where each entry of $\mathbf{A}$ can take an arbitrary value in the interval defined by the corresponding entry of $[\mathbf{A}]$.

Remark 3: Equation (25) can be used to represent general uMPL systems, including autonomous and nonautonomous uMPL systems. For instance, in order to represent an autonomous uMPL system, consider $p=n, \mathbf{z} \equiv \mathbf{x}(k), \mathbf{x} \equiv$ $\mathbf{x}(k-1)$ and $\mathbf{A} \equiv \mathbf{A}(k)$, see equation (18).

\section{A. The Image of a Point}

Let equation (25) be an uMPL system. If the point $\mathbf{x}$ is given, then $\mathbf{z} \in[\mathbf{z}]$ where:

$$
[\mathbf{z}]=[\mathbf{A}] \otimes \mathbf{x} .
$$

From (26), (13) and (14), it can be demonstrated that $z_{i}$ is in the interval defined by:

$$
\left[z_{i}\right]=\left[\bigoplus_{j=1}^{p}\left(\underline{a}_{i j} \otimes x_{j}, \geq\right), \bigoplus_{j=1}^{p}\left(\bar{a}_{i j} \otimes x_{j}, \leq\right)\right] .
$$

Therefore, the image of a point $\mathrm{x}$ w.r.t. the uMPL system is the hyperrectangle given by:

$$
\mathcal{I}_{[\mathbf{A}]}\{\mathbf{x}\}=\bigcap_{i=1}^{n}\left\{\mathbf{z} \in \mathbb{R}^{n}: \bigoplus_{j=1}^{p} \underline{a}_{i j} \otimes x_{j} \preceq z_{i} \preceq \bigoplus_{j=1}^{p} \bar{a}_{i j} \otimes x_{j}\right\},
$$

or equivalently,

$$
\mathcal{I}_{[\mathbf{A}]}\{\mathbf{x}\}=\left\{\mathbf{z} \in \mathbb{R}^{n}: \underline{\mathbf{A}} \otimes \mathbf{x} \preceq \mathbf{z} \preceq \overline{\mathbf{A}} \otimes \mathbf{x}\right\} .
$$

Thus, the image of a given point can be promptly computed by using (26) or (27).

Example 3: Consider the uMPL system of Example 2. From (27), given $\mathbf{x}(0)=\left(\begin{array}{ll}1 & 1\end{array}\right)^{T}$, we have that

$$
[\mathbf{x}](1)=[\mathbf{A}] \otimes \mathbf{x}(0)=\left(\begin{array}{ll}
{[(3, \geq),} & (4, \leq)] \\
{[(4, \geq),} & (7, \leq)]
\end{array}\right) .
$$

Therefore, $\mathcal{I}_{[\mathbf{A}]}\{\mathbf{x}(0)\}=\left\{\mathbf{x} \in \mathbb{R}^{2}: 3 \leq x_{1} \leq 4,4 \leq x_{2} \leq 7\right\}$.

\section{B. The Inverse Image of a Point}

The computation of the inverse image of a given point depicted by vector $\mathbf{z}$ w.r.t. an UMPL system is now addressed, this task is not as straightforward as the direct image computation. Let equation (25) be an UMPL system, then the inverse image of $\mathbf{z}$ is defined as:

$$
\mathcal{I}_{[\mathbf{A}]}^{-1}\{\mathbf{z}\}=\left\{\mathbf{x} \in \mathbb{R}^{p}: \exists \mathbf{A} \in[\mathbf{A}]: \mathbf{A} \otimes \mathbf{x}=\mathbf{z}\right\} .
$$

Lemma 1: Given $\mathbf{x}$ and $\mathbf{z}$, if $\underline{\mathbf{A}} \otimes \mathbf{x} \preceq \mathbf{z} \preceq \overline{\mathbf{A}} \otimes \mathbf{x}$ then, there exists $\mathbf{A} \in[\mathbf{A}]$ (i.e., $\underline{\mathbf{A}} \preceq \mathbf{A} \preceq \overline{\mathbf{A}}$ ) such that $\mathbf{A} \otimes \mathbf{x}=\mathbf{z}$.

Proof: The greatest $\mathbf{A}$ that satisfies $\mathbf{A} \otimes \mathbf{x} \preceq \mathbf{z}$ is given by $\hat{\mathbf{A}}=\mathbf{z} \phi \mathbf{x}$ (see (22)). Therefore, $\underline{\mathbf{A}} \otimes \mathbf{x} \preceq \mathbf{z} \Rightarrow \underline{\mathbf{A}} \preceq \hat{\mathbf{A}}$. In 
addition, since $\underline{\mathbf{A}} \preceq \overline{\mathbf{A}}: \underline{\mathbf{A}} \preceq \hat{\mathbf{A}} \wedge \overline{\mathbf{A}} \preceq \overline{\mathbf{A}}$. Now, let us consider $\mathbf{z}^{\prime}=(\hat{\mathbf{A}} \wedge \overline{\mathbf{A}}) \otimes \mathbf{x}$, from (22), $\hat{a}_{i j}=z_{i}-x_{j}$ and, from (7):

$$
\begin{aligned}
z_{i}^{\prime} & =\bigoplus_{k=1}^{m}\left\{\left[\left(z_{i}-x_{k}\right) \wedge \bar{a}_{i k}\right] \otimes x_{k}\right\}=\bigoplus_{k=1}^{m}\left\{\left[z_{i} \wedge\left(\bar{a}_{i k} \otimes x_{k}\right)\right]\right\} \\
& =z_{i} \wedge \bigoplus_{k=1}^{m}\left[\bar{a}_{i k} \otimes x_{k}\right] .
\end{aligned}
$$

Hence, $\mathbf{z}^{\prime}=\mathbf{z} \wedge \overline{\mathbf{A}} \otimes \mathbf{x}$. By assumption, $\mathbf{z} \preceq \overline{\mathbf{A}} \otimes \mathbf{x}$, therefore, $\mathbf{z}^{\prime}=\mathbf{z}$. Hence, if $\underline{\mathbf{A}} \otimes \mathbf{x} \preceq \mathbf{z} \preceq \overline{\mathbf{A}} \otimes \mathbf{x}$ the matrix $(\hat{\mathbf{A}} \wedge \overline{\mathbf{A}}) \in[\mathbf{A}]$ is such that $(\hat{\mathbf{A}} \wedge \overline{\mathbf{A}}) \otimes \mathbf{x}=\mathbf{z}$.

Example 4: Consider the matrix $[\mathbf{A}]$ of Example 2. Given $\mathbf{x}(0)=\left(\begin{array}{ll}1 & 1\end{array}\right)^{T}$ and $\mathbf{x}(1)=\left(\begin{array}{lll}3.3 & 4.8\end{array}\right)^{T}$, note that $\underline{\mathbf{A}} \otimes \mathbf{x}(0) \preceq$ $\mathbf{x}(1) \preceq \overline{\mathbf{A}} \otimes \mathbf{x}(0)$. Then, form Lemma 1, there exists $\mathbf{A}(1) \in$ $[\mathbf{A}]$ such that $\mathbf{A}(1) \otimes \mathbf{x}(0)=\mathbf{x}(1)$. Moreover, the greatest $\mathbf{A}(1) \in[\mathbf{A}]$ that satisfies this expression is given by

$$
\hat{\boldsymbol{A}} \wedge \overline{\boldsymbol{A}}=\left(\begin{array}{cc}
2.3 & 2 \\
3.8 & 3.8
\end{array}\right), \text { where } \hat{\boldsymbol{A}}=\mathbf{x}(1) \phi \mathbf{x}(0) .
$$

Proposition 1: A point $\mathrm{x}$ is in the inverse image of $\mathrm{z}$ w.r.t. an uMPL system if and only if $\mathbf{z}$ is in the image of $\mathbf{x}$ w.r.t. the uMPL system, i.e.,

$$
\mathbf{x} \in \mathcal{I}_{[\mathbf{A}]}^{-1}\{\mathbf{z}\} \Leftrightarrow \underline{\mathbf{A}} \otimes \mathbf{x} \preceq \mathbf{z} \preceq \overline{\mathbf{A}} \otimes \mathbf{x} .
$$

Proof: From (30) if $\mathbf{x} \in \mathcal{I}_{[\mathbf{A}]}^{-1}\{\mathbf{z}\} \Rightarrow \underline{\mathbf{A}} \otimes \mathbf{x} \preceq \mathbf{z} \preceq \overline{\mathbf{A}} \otimes \mathbf{x}$, since $\mathbf{A} \in[\mathbf{A}]$. Lemma 1 yields that if $\underline{\mathbf{A}} \otimes \mathbf{x} \preceq \mathbf{z} \preceq \overline{\mathbf{A}} \otimes \mathbf{x}$ there is $\mathbf{A} \in[\mathbf{A}]$ such that $\mathbf{A} \otimes \mathbf{x}=\mathbf{z}$, i.e., $\mathbf{x} \in \mathcal{I}_{[\mathbf{A}]}^{-1}\{\mathbf{z}\}$, hence $\underline{\mathbf{A}} \otimes \mathbf{x} \preceq \mathbf{z} \preceq \overline{\mathbf{A}} \otimes \mathbf{x} \Rightarrow \mathbf{x} \in \mathcal{I}_{[\mathbf{A}]}^{-1}\{\mathbf{z}\}$.

From Proposition 1, it appears that a point $\mathrm{x}$ belongs to the inverse image of a point $\mathbf{z}$ if and only if the following inequalities are true:

$$
\begin{aligned}
& \underline{\mathbf{A}} \otimes \mathbf{x} \preceq \mathbf{z}, \\
& \overline{\mathbf{A}} \otimes \mathbf{x} \succeq \mathbf{z} .
\end{aligned}
$$

Thus, the inverse image of a point $\mathbf{z}$ can be represented by the intersection of two sets:

$$
\mathcal{I}_{[\mathbf{A}]}^{-1}\{\mathbf{z}\}=U \cap L
$$

where $U$ is the set of all $\mathbf{x}$ that satisfies (31) and $L$ is the set of all $\mathrm{x}$ that satisfies (32).

By using residuation, see (21), the set $U$ is given by:

$$
\begin{aligned}
U & =\left\{\mathbf{x} \in \mathbb{R}^{p}: \underline{\mathbf{A}} \otimes \mathbf{x} \preceq \mathbf{z}\right\} \\
& =\left\{\mathbf{x} \in \mathbb{R}^{p}: \mathbf{x} \preceq \underline{\mathbf{A}} \phi \mathbf{z}\right\} .
\end{aligned}
$$

The set $L$ is given by:

$$
\begin{aligned}
L & =\left\{\mathbf{x} \in \mathbb{R}^{p}: \overline{\mathbf{A}} \otimes \mathbf{x} \succeq \mathbf{z}\right\} \\
& =\bigcap_{i=1}^{n}\left\{\mathbf{x} \in \mathbb{R}^{p}: z_{i} \preceq \bigoplus_{j=1}^{p} \bar{a}_{i j} \otimes x_{j}\right\} .
\end{aligned}
$$

In order to obtain a representation of $L$ that can be represented by interval matrices, some mathematical manipulation are required. First, recall that, according to De Morgan's Laws, the complement of the intersection of $n$ sets and the complement of the union of $n$ sets are, respectively, given by

$$
\left(\bigcap_{j=1}^{n} \mathcal{A}_{j}\right)^{c}=\bigcup_{j=1}^{n} \mathcal{A}_{j}^{c}, \quad\left(\bigcup_{j=1}^{n} \mathcal{A}_{j}\right)^{c}=\bigcap_{j=1}^{n} \mathcal{A}_{j}^{c} .
$$

An alternative equation to compute the complement of the intersection is given by:

$$
\begin{aligned}
\left(\bigcap_{j=1}^{n} \mathcal{A}_{j}\right)^{c} & =\mathcal{A}_{1}^{c} \cup\left[\mathcal{A}_{1} \cap \mathcal{A}_{2}^{c}\right] \cup \cdots \cup\left[\left(\bigcap_{k=1}^{n-1} \mathcal{A}_{k}\right) \cap \mathcal{A}_{n}^{c}\right] \\
& =\bigcup_{j=1}^{n}\left[\left(\bigcap_{k=1}^{j-1} \mathcal{A}_{k}\right) \cap \mathcal{A}_{j}^{c}\right]
\end{aligned}
$$

where $\bigcap_{k=1}^{0} \mathcal{A}_{k}=\mathbb{R}^{p}$.

Note that, from (36), the complement of the intersection of sets can be represented by a union of sets and, from (37), the complement of the intersection can be represented by a union of pairwise disjoint sets. As it will be demonstrated in this section, equation (37) plays an important role in the handling of set $L$ since it yields a representation defined by the union of pairwise disjoint sets, which reduces the computational effort to compute the inverse image of a point.

Example 5: Let us compute the complement of the set $\left\{\mathbf{x} \in \mathbb{R}^{3}: \mathbf{x}<(5,2,4)^{T}\right\}$. This set can be expressed as $\underbrace{\left\{\mathbf{x} \in \mathbb{R}^{3}: x_{1}<5\right\}}_{\mathcal{A}_{1}} \cap \underbrace{\left\{\mathbf{x} \in \mathbb{R}^{3}: x_{2}<2\right\}}_{\mathcal{A}_{2}} \cap \underbrace{\left\{\mathbf{x} \in \mathbb{R}^{3}: x_{3}<4\right\}}_{\mathcal{A}_{3}}$. According to (36): $\left\{\mathbf{x} \in \mathbb{R}^{3}: \mathbf{x}<(5,2,4)^{T}\right\}^{c}=$ $\left\{\mathbf{x} \in \mathbb{R}^{3}: x_{1} \geq 5\right\} \cup\left\{\mathbf{x} \in \mathbb{R}^{3}: x_{2} \geq 2\right\} \cup\left\{\mathbf{x} \in \mathbb{R}^{3}: x_{3} \geq 4\right\}$, which is the union of non pairwise disjoint sets. And, according to (37): $\left\{\mathbf{x} \in \mathbb{R}^{3}: \mathbf{x}<(5,2,4)^{T}\right\}^{c}=\left\{\mathbf{x} \in \mathbb{R}^{3}\right.$ : $\left.x_{1} \geq 5\right\} \cup\left(\left\{\mathbf{x} \in \mathbb{R}^{3}: x_{1}<5\right\} \cap\left\{\mathbf{x} \in \mathbb{R}^{3}: x_{2} \geq 2\right\}\right) \cup(\{\mathbf{x} \in$ $\left.\left.\mathbb{R}^{3}: x_{1}<5\right\} \cap\left\{\mathbf{x} \in \mathbb{R}^{3}: x_{2}<2\right\} \cap\left\{\mathbf{x} \in \mathbb{R}^{3}: x_{3} \geq 4\right\}\right)$, which is the union of pairwise disjoint sets.

From (36), the complement of $L$ is given by:

$$
\begin{aligned}
L^{c} & =\left(\bigcap_{i=1}^{n}\left\{\mathrm{x} \in \mathbb{R}^{p}: z_{i} \preceq \bigoplus_{j=1}^{p} \bar{a}_{i j} \otimes x_{j}\right\}\right)^{c} \\
& =\bigcup_{i=1}^{n}\left\{\mathrm{x} \in \mathbb{R}^{p}: z_{i} \preceq \bigoplus_{j=1}^{p} \bar{a}_{i j} \otimes x_{j}\right\} .
\end{aligned}
$$

Since $z_{i}$ is scalar, $\left\{\mathbf{x} \in \mathbb{R}^{p}: z_{i} \preceq \bigoplus_{j=1}^{p} \bar{a}_{i j} \otimes x_{j}\right\}^{c}=$ $\left\{\mathbf{x} \in \mathbb{R}^{p}: z_{i} \succ \bigoplus_{j=1}^{p} \bar{a}_{i j} \otimes x_{j}\right\}$. Thus,

$$
L^{c}=\bigcup_{i=1}^{n}\left\{\mathrm{x} \in \mathbb{R}^{p}: \bigoplus_{j=1}^{p} \bar{a}_{i j} \otimes x_{j} \prec z_{i}\right\}
$$

Lemma 2: The set $L^{c}$ can be expressed by:

$$
L^{c}=\bigcup_{i=1}^{n}\left\{\mathbf{x} \in \mathbb{R}^{p}: \mathbf{x}<\underline{\mathbf{x}}^{(i)}\right\}
$$

where $\underline{\mathbf{X}}^{(i)}$ is a vector with entries defined by $\underline{\mathbf{X}}_{j}^{(i)}=z_{i}-\bar{a}_{i j}$. 
Proof: Observe that:

$$
\bigoplus_{j=1}^{p} \bar{a}_{i j} \otimes x_{j} \prec z_{i} \Leftrightarrow\left(\forall j, x_{j}<z_{i}-\bar{a}_{i j}=\underline{\mathbf{x}}_{j}^{(i)}\right) .
$$

Therefore, $\bigoplus_{j=1}^{p} \bar{a}_{i j} \otimes x_{j} \prec z_{i} \Leftrightarrow \mathbf{x}<\underline{\mathbf{X}}^{(i)}$.

Each term of the union that represents $L^{c}$ in (39) can be expressed as an intersection of sets as follows:

$$
\begin{aligned}
\left\{\mathbf{x} \in \mathbb{R}^{p}: \mathbf{x}<\underline{\mathbf{X}}^{(i)}\right\} & =\left\{\mathbf{x} \in \mathbb{R}^{p}: x_{j}<\underline{\mathbf{X}}_{j}^{(i)} \forall j \in\{1, \ldots, p\}\right\} \\
& =\bigcap_{j=1}^{p}\left\{\mathbf{x} \in \mathbb{R}^{p}: x_{j}<\underline{\mathbf{x}}_{j}^{(i)}\right\} .
\end{aligned}
$$

Therefore, equation (39) can be expressed as:

$$
L^{c}=\bigcup_{i=1}^{n}\left(\bigcap_{j=1}^{p}\left\{\mathbf{x} \in \mathbb{R}^{p}: x_{j}<\underline{\mathbf{x}}_{j}^{(i)}\right\}\right) .
$$

Notation 3: The sets $U$ and $L$ are defined by unions and intersections of subsets defined by inequalities. In the following, it will be used a short notation to represent this subsets, in which these inequalities appear in braces $\{\cdot\}$. For instance, the set $\left\{\mathrm{x} \in \mathbb{R}^{p}: x_{j}<\underline{\mathbf{X}}_{j}^{(i)}\right\}$ is represented by $\left\{x_{j}<\underline{\mathbf{X}}_{j}^{(i)}\right\}$. Note that, this notation will be used when it is clear that $\mathbf{x} \in \mathbb{R}^{p}$, since this information is omitted.

The set $L$ can be obtained by computing the complement of $L^{c}$, i.e., $L=\left(L^{c}\right)^{c}$. Thus, the set $L$ is given by (see (36)):

$$
L=\left[\bigcup_{i=1}^{n}\left(\bigcap_{j=1}^{p}\left\{x_{j}<\underline{\mathbf{x}}_{j}^{(i)}\right\}\right)\right]^{c}=\bigcap_{i=1}^{n}\left[\left(\bigcap_{j=1}^{p}\left\{x_{j}<\underline{\mathbf{x}}_{j}^{(i)}\right\}\right)^{c}\right] .
$$

According to (36),

$$
\left(\bigcap_{j=1}^{p}\left\{x_{j}<\underline{\mathbf{x}}_{j}^{(i)}\right\}\right)^{c}=\bigcup_{j=1}^{p}\left\{x_{j}<\underline{\mathbf{x}}_{j}^{(i)}\right\}^{c}=\bigcup_{j=1}^{p}\left\{x_{j} \geq \underline{\mathbf{x}}_{j}^{(i)}\right\} \text {. }
$$

Thus,

$$
L=\bigcap_{i=1}^{n}\left(\bigcup_{j=1}^{p}\left\{x_{j} \geq \underline{\mathbf{x}}_{j}^{(i)}\right\}\right)
$$

From (43) it can be shown that the set $L$ can be represented by a union of $N=p^{n}$ hyperrectangles. In order to reduce the computational effort, in the following it is shown that if we consider (37) instead of (36) to compute the complement of an intersection of sets, the set $L$ can be represented by a set of $N^{\prime} \leq N$ pairwise disjoint hyperrectangles.

According to (37), equation (42) becomes:

$$
\left(\bigcap_{j=1}^{p}\left\{x_{j}<\underline{\mathbf{X}}_{j}^{(i)}\right\}\right)^{c}=\bigcup_{j=1}^{p}\left[\left(\bigcap_{k=1}^{j-1}\left\{x_{k}<\underline{\mathbf{X}}_{k}^{(i)}\right\}\right) \cap\left\{x_{j} \geq \underline{\mathbf{X}}_{j}^{(i)}\right\}\right]
$$

where $\mathbf{x} \in \mathbb{R}^{p}$ and $\bigcap_{k=1}^{0}\left\{x_{k}<\underline{\mathbf{X}}_{k}^{(i)}\right\}=\mathbb{R}^{p}$.

From (41) and (44):

$$
L=\bigcap_{i=1}^{n}\left(\bigcup_{j=1}^{p}\left[\left(\bigcap_{k=1}^{j-1}\left\{x_{k}<\underline{\mathbf{x}}_{k}^{(i)}\right\}\right) \cap\left\{x_{j} \geq \underline{\mathbf{x}}_{j}^{(i)}\right\}\right]\right)
$$

Defining:

$$
\operatorname{set}_{j}^{i}=\left[\bigcap_{k=1}^{j-1}\left\{x_{k}<\underline{\mathbf{x}}_{k}^{(i)}\right\}\right] \cap\left\{x_{j} \geq \underline{\mathbf{x}}_{j}^{(i)}\right\}
$$

we have that:

$$
\begin{aligned}
& L=\bigcap_{i=1}^{n} \bigcup_{j=1}^{p} s e t_{j}^{i} \\
& =\left(\operatorname{set}_{1}^{1} \cup \cdots \cup \operatorname{set}_{p}^{1}\right) \cap\left(\operatorname{set}_{1}^{2} \cup \cdots \cdots \cup \operatorname{set}_{p}^{2}\right) \cap \\
& \cdots \cap\left(\operatorname{set}_{1}^{n} \cup \cdots \cup \operatorname{set}_{p}^{n}\right) \\
& =\left(\operatorname{set}_{1}^{1} \cap \operatorname{set}_{1}^{2} \cap \cdots \cap \operatorname{set}_{1}^{n}\right) \cup\left(\operatorname{set}_{1}^{1} \cap \operatorname{set}_{1}^{2} \cap \cdots \cap \operatorname{set}_{2}^{n}\right) \\
& \cdots \cup\left(\operatorname{set}_{p}^{1} \cap \operatorname{set}_{p}^{2} \cap \cdots \cap \operatorname{set}_{p}^{n}\right)
\end{aligned}
$$

In order to obtain a compact representation of (47), the set of coefficients $\mathcal{G}$ is defined as:

$$
\mathcal{G}=\{1, \ldots, p\}^{n} .
$$

Remark 4: If $\mathbf{g} \in \mathcal{G}$, then $\mathbf{g}=\left(g_{1}, g_{2}, \cdots, g_{n}\right)$, where $g_{i} \in\{1, \cdots, p\}$.

Thus, equation (47) can be rewritten as:

$$
L=\bigcup_{\mathbf{g} \in \mathcal{G}} \bigcap_{i=1}^{n} s e t_{g_{i}}^{i} .
$$

Now, let us define:

$$
S E T^{\mathbf{g}}=\bigcap_{i=1}^{n} \operatorname{set}_{g_{i}}^{i} .
$$

Lemma 3: The sets $S E T^{\mathbf{g}}$ for $\mathbf{g} \in \mathcal{G}$ are pairwise disjoint hyperrectangles.

Proof: Consider $\mathbf{g}$ and $\mathbf{g}^{\prime} \in \mathcal{G}$ with $\mathbf{g} \neq \mathbf{g}^{\prime}$. In this case, there is $k \in\{1, \ldots, n\}$ such that $g_{k} \neq g_{k}^{\prime}$. From (50),

$$
\begin{aligned}
S E T^{\mathbf{g}} \cap S E T^{\mathbf{g}^{\prime}} & =\bigcap_{i=1}^{n} \operatorname{set}_{g_{i}}^{i} \cap \bigcap_{i=1}^{n} \operatorname{set}_{g_{i}^{\prime}}^{i} \\
& =\left[\bigcap_{\substack{i=1 \\
i \neq k}}^{n}\left(\operatorname{set}_{g_{i}}^{i} \cap \operatorname{set}_{g_{i}^{\prime}}^{i}\right)\right] \cap\left[\operatorname{set}_{g_{k}}^{k} \cap \operatorname{set}_{g_{k}^{\prime}}^{k}\right] .
\end{aligned}
$$

Note that, according (37), the sets $\left\{\right.$ set $_{1}^{i}, \ldots$, set $\left._{p}^{i}\right\}$, with set $_{j}^{i}$ defined by (46), are pairwise disjoint for a fixed $i$. Therefore, since $g_{k} \neq g_{k}^{\prime}$, we have that set $g_{g_{k}}^{k} \cap$ set $_{g_{k}^{\prime}}^{k}=\emptyset$ and hence $S E T^{\mathbf{g}} \cap S E T^{\mathbf{g}^{\prime}}=\emptyset$.

From (49) and (50), the set $L$ can be expressed as:

$$
L=\bigcup_{\mathbf{g} \in \mathcal{G}} S E T^{\mathbf{g}}
$$

Remark 5: In the worst-case scenario the set $L$, given by (51), can be represented by $N^{\prime}=p^{n}$ pairwise disjoint hyperrectangles. However, some $S E T^{\mathrm{g}}$ may be empty. Hence, in general, the set $L$ can be represented by $N^{\prime} \leq p^{n}$ pairwise disjoint hyperrectangles.

Then, from (33), we have that:

$$
\mathcal{I}_{[\mathbf{A}]}^{-1}\{\mathbf{z}\}=U \cap \bigcup_{\mathbf{g} \in \mathcal{G}} S E T^{\mathbf{g}}=\bigcup_{\mathbf{g} \in \mathcal{G}}\left(S E T^{\mathbf{g}} \cap U\right),
$$

where $U$ is defined by (34). 
Example 6: Consider the autonomous uMPL system given by:

$$
\mathbf{x}(k)=\mathbf{A}(k) \otimes \mathbf{x}(k-1),
$$

where,

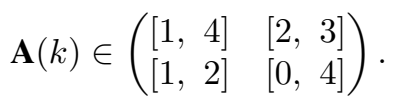

Given $\mathbf{x}(1)=(5,4)^{T}$, let us compute $\mathcal{I}_{[\mathbf{A}]}^{-1}\{\mathbf{x}(1)\}$. According to (34), the set $U$ is given by:

$U=\left\{\mathbf{x} \in \mathbb{R}^{2}: \mathbf{x} \preceq \underline{\mathbf{A}} \phi \mathbf{x}(1)\right\}$, where,

$\underline{\mathbf{A}} \phi \mathbf{x}(1)=\left(\begin{array}{ll}1 & 2 \\ 1 & 0\end{array}\right) \phi\left(\begin{array}{l}5 \\ 4\end{array}\right)=\left(\begin{array}{l}(5-1) \wedge(4-1) \\ (5-2) \wedge(4-0)\end{array}\right)=\left(\begin{array}{l}3 \\ 3\end{array}\right)$.

Thus, $U=\left\{\mathbf{x} \in \mathbb{R}^{2}: x_{1} \leq 3, x_{2} \leq 3\right\}$. In order to compute the sets $\operatorname{set}_{j}^{i}, i, j \in\{1,2\}$, we must compute first $\underline{\mathbf{X}}^{(i)}$ for $i \in\{1,2\}$ (see (39)):

$$
\begin{aligned}
& \underline{\mathbf{X}}^{(1)}=\left(\begin{array}{l}
x_{1}(1)-\bar{a}_{11} \\
x_{1}(1)-\bar{a}_{12}
\end{array}\right)=\left(\begin{array}{l}
(5-4) \\
(5-3)
\end{array}\right)=\left(\begin{array}{l}
1 \\
2
\end{array}\right), \\
& \underline{\mathbf{X}}^{(2)}=\left(\begin{array}{l}
x_{2}(1)-\bar{a}_{21} \\
x_{2}(1)-\bar{a}_{22}
\end{array}\right)=\left(\begin{array}{l}
(4-2) \\
(4-4)
\end{array}\right)=\left(\begin{array}{l}
2 \\
0
\end{array}\right) .
\end{aligned}
$$

According to (46), the sets $\operatorname{set}_{j}^{i}, i, j \in\{1,2\}$, are given by:

set $_{1}^{1}=\left\{\mathbf{x} \in \mathbb{R}^{2}: x_{1} \geq 1\right\}, \quad$ set $_{2}^{1}=\left\{\mathbf{x} \in \mathbb{R}^{2}: x_{1}<1, x_{2} \geq 2\right\}$

set $_{1}^{2}=\left\{\mathbf{x} \in \mathbb{R}^{2}: x_{1} \geq 2\right\}, \quad \operatorname{set}_{2}^{2}=\left\{\mathbf{x} \in \mathbb{R}^{2}: x_{1}<2, x_{2} \geq 0\right\}$

Now, for each $\mathbf{g} \in\{1,2\}^{2}=\{(1,1),(1,2),(2,1),(2,2)\}$ we compute the sets $S E T^{\mathrm{g}}$ as follows:

$S E T^{(1,1)}=\operatorname{set}_{1}^{1} \cap \operatorname{set}_{1}^{2}=\left\{\mathbf{x} \in \mathbb{R}^{2}: x_{1} \geq 2\right\}$,

$S E T^{(1,2)}=\operatorname{set}_{1}^{1} \cap \operatorname{set}_{2}^{2}=\left\{\mathbf{x} \in \mathbb{R}^{2}: 1 \leq x_{1}<2, x_{2} \geq 0\right\}$,

$S E T^{(2,1)}=\operatorname{set}_{2}^{1} \cap \operatorname{set}_{1}^{2}=\emptyset$,

$S E T^{(2,2)}=\operatorname{set}_{2}^{1} \cap \operatorname{set}_{2}^{2}=\left\{\mathbf{x} \in \mathbb{R}^{2}: x_{1}<1, x_{2} \geq 2\right\}$.

Finally we compute $\mathcal{I}_{[\mathbf{A}]}^{-1}\{\mathbf{x}(1)\}=\bigcup_{\mathbf{g} \in\{1, \ldots, p\}^{n}}\left(S E T^{\mathbf{g}} \cap U\right)$ (see (52)):

$$
\begin{aligned}
& \mathcal{I}_{[\mathbf{A}]}^{-1}\{\mathbf{x}(1)\}=\left\{\mathbf{x} \in \mathbb{R}^{2}: 2 \leq x_{1} \leq 3, x_{2} \leq 3\right\} \\
& \cup\left\{\mathbf{x} \in \mathbb{R}^{2}: 1 \leq x_{1}<2,0 \leq x_{2} \leq 3\right\} \\
& \cup\left\{\mathbf{x} \in \mathbb{R}^{2}: x_{1}<1,2 \leq x_{2} \leq 3\right\} .
\end{aligned}
$$

The inverse image of $\mathbf{x}(1)$ can be observed in Figure 1. Note that $\mathcal{I}_{[\mathbf{A}]}^{-1}\{\mathbf{x}(1)\}$ is a union of pairwise disjoint hyperrectangles.

Remark 6: The inverse image of a point $\mathbf{z}$ w.r.t. an uMPL system may not exist, in this case, there is no $\mathbf{x}$ such that $\mathbf{z} \in[\mathbf{A}] \otimes \mathbf{x}$ and therefore (33) will return the empty set.

In the next section, it is presented an algorithm to compute the inverse image of a point based on the interval matrices.

\section{An algorithm to compute $\mathcal{I}_{[\mathbf{A}]}^{-1}\{\mathbf{z}\}$}

First, note that (52) can be expressed as:

$$
\begin{aligned}
\mathcal{I}_{[\mathbf{A}]}^{-1}\{\mathbf{z}\} & =\bigcup_{\mathbf{g} \in \mathcal{G}}\left(S E T^{\mathbf{g}} \cap U\right) \\
& =\bigcup_{\mathbf{g} \in \mathcal{G}} \bigcap_{i=1}^{n}\left(\operatorname{set}_{g_{i}}^{i} \cap U\right)=\bigcup_{\mathbf{g} \in \mathcal{G}} S E T^{\mathbf{g}} U,
\end{aligned}
$$

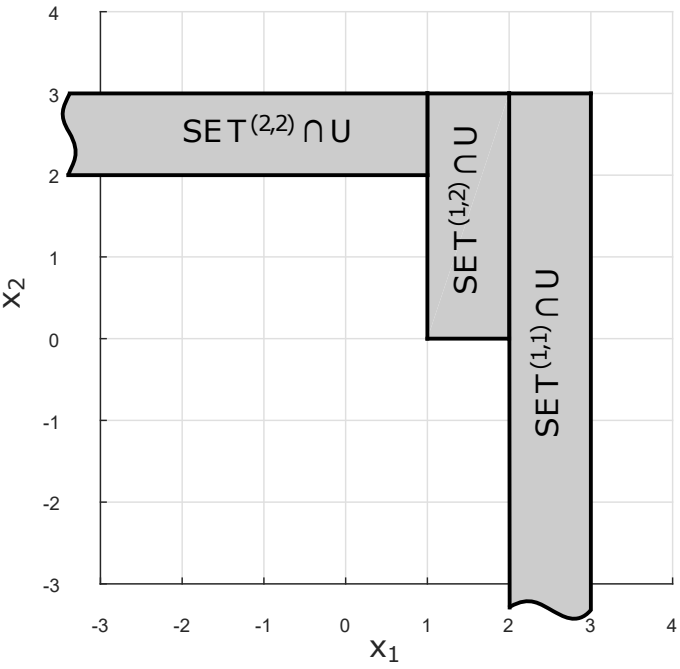

Fig. 1. Inverse image of $\mathbf{x}(1)$

where $S E T^{\mathbf{g}} U=\bigcap_{i=1}^{n}\left(\right.$ set $\left._{g_{i}}^{i} \cap U\right)$.

The set $U$, given by (34), can be represented by:

$$
[\mathbf{U}]=\left(\begin{array}{cc}
{[(-\infty,>),} & \left.\left(\overline{\mathbf{X}}_{1}, \leq\right)\right] \\
\vdots & \\
{[(-\infty,>),} & \left.\left(\overline{\mathbf{X}}_{p}, \leq\right)\right]
\end{array}\right) .
$$

where $\overline{\mathbf{X}}=\underline{\mathbf{A}} \phi \mathbf{z}$.

From (46),

$$
\operatorname{set}_{j}^{i} \cap U=\left[\bigcap_{k=1}^{j-1}\left\{x_{k}<\underline{\mathbf{x}}_{k}^{(i)}\right\}\right] \cap\left\{x_{j} \geq \underline{\mathbf{x}}_{j}^{(i)}\right\} \cap U .
$$

Thus, the intersection $\operatorname{set}_{j}^{i} \cap U$ can be represented by the following $(p \times 1)$ interval matrix:

$$
\left[\operatorname{set}_{\mathbf{j}}^{\mathrm{i}} \cap \mathbf{U}\right]=\left(\begin{array}{c}
{\left[(-\infty,>), \min \left\{\left(\underline{\mathbf{x}}_{1}^{(i)},<\right),\left(\overline{\mathbf{x}}_{1}, \leq\right)\right\}\right]} \\
\vdots \\
{\left[(-\infty,>), \min \left\{\left(\underline{\mathbf{x}}_{j-1}^{(i)},<\right),\left(\overline{\mathbf{x}}_{j-1}, \leq\right)\right\}\right]} \\
{\left[\left(\underline{\mathbf{x}}_{j}^{(i)}, \geq\right),\left(\overline{\mathbf{x}}_{j}, \leq\right)\right]} \\
{\left[(-\infty,>),\left(\overline{\mathbf{x}}_{j+1}, \leq\right)\right]} \\
\vdots \\
{\left[(-\infty,>),\left(\overline{\mathbf{x}}_{p}, \leq\right)\right]}
\end{array}\right) .
$$

Remark 7: Note that, whenever $\underline{\mathbf{X}}_{j}^{(i)}>\overline{\mathbf{X}}_{j}$, the intersection set $_{j}^{i} \cap U$ will represent an empty region (see section II-A). Unnecessary computations can be avoided by taking this fact into account.

After computing $\left[\operatorname{set}_{\mathbf{j}}^{\mathbf{i}} \cap \mathbf{U}\right]$ for all $i$ and $j$, the sets $S E T^{\mathbf{g}} U$ can be computed by:

$$
\left[\operatorname{SET}^{\mathbf{g}} \mathbf{U}\right]=\bigcap_{i=1}^{n}\left[\operatorname{set}_{\mathbf{g}_{\mathbf{i}}}^{\mathbf{i}} \cap \mathbf{U}\right] .
$$

Thus, the inverse image of a point can be represented by

$$
\mathcal{I}_{[\mathbf{A}]}^{-1}\{\mathbf{z}\}=\bigcup_{\mathbf{g} \in \mathcal{G}}\left[\mathbf{S E T}^{\mathbf{g}} \mathbf{U}\right] .
$$


Thus the inverse image of a point w.r.t. an UMPL system can be completely represented by the union of pairwise disjoint hyperrectangles.

Algorithm 1 describes a general procedure for computing (58). The inputs of the algorithm are the point $\mathbf{z}$ and system interval matrix $[\mathbf{A}]$, the output is the collection of hyperrectangles representing the inverse image of $\mathbf{z}$. The worstcase complexity of the Algorithm is computed as follows: the worst-case complexity of step 10 is $\mathcal{O}\left(p^{n}\right)$ and the complexity of steps 11 to 13 amounts to $\mathcal{O}(n p)$. Therefore, the worst-case complexity of the algorithm is $\mathcal{O}\left(n p^{n+1}\right)$.

Remark 8: It is recalled that the worst-case complexity to compute the inverse image of a point w.r.t. an uMPL system using the DBM approach is $\mathcal{O}\left(p^{n}(n+p)^{3}\right)$ [27], [28].

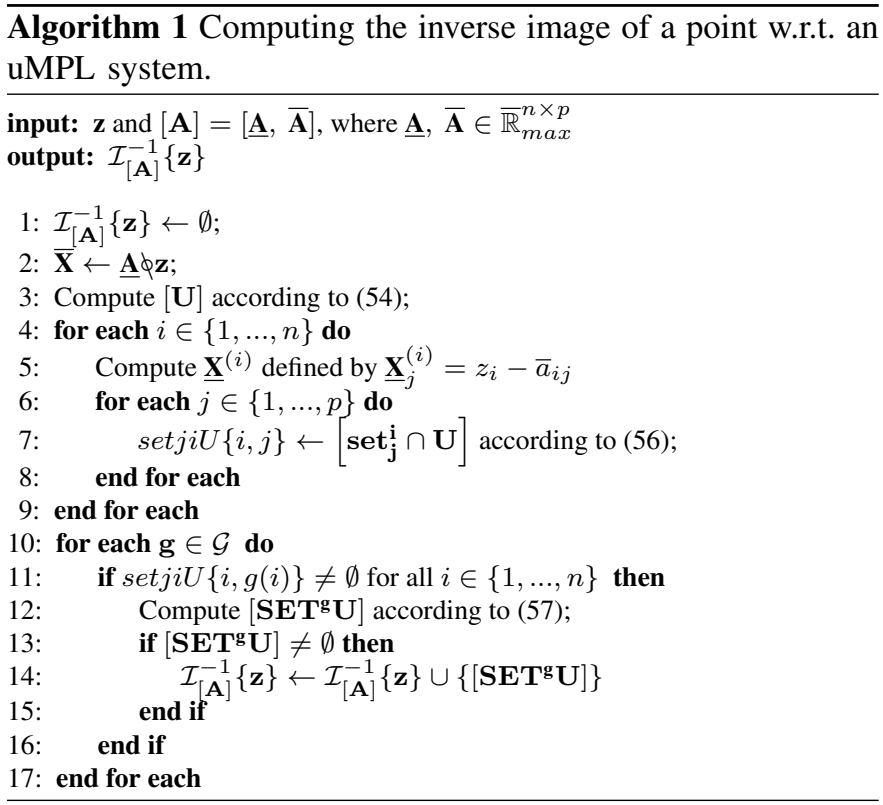

\section{Applichtion to a Filtering Problem}

This section deals with the solution of a filtering problem. The procedures for computing the image and the inverse image of a point, presented in this paper, are applied to compute and represent the support of the posterior probability density function (p.d.f) of the system states as a union of hyperrectangles. The obtained hyperrectangles are then efficiently used to solve the filtering problem.

Problem 1: Consider the UMPL system given by:

$$
\begin{aligned}
& \mathbf{x}(k)=\mathbf{A}(k) \otimes \mathbf{x}(k-1), \mathbf{A}(k) \in\left(\begin{array}{cc}
{\left[\begin{array}{ll}
1 & 3
\end{array}\right]} & {\left[\begin{array}{ll}
0 & 4
\end{array}\right]} \\
{[2} & 4
\end{array}\right] \\
& \left.\mathbf{z}(k)=\mathbf{C}(k) \otimes \mathbf{x}(k), \mathbf{C}(k) \in\left(\begin{array}{lll}
0.5 & {[0} & 1
\end{array}\right]\right) .
\end{aligned}
$$

In addition, consider that the nondeterministic matrices entries are random variables uniformly distributed in the given intervals, e.g., the element $a_{12}$ of $\mathbf{A}(k)$ is uniformly distributed between 0 and 4 . Given $\mathbf{x}(0)=(0,0)^{T}$ and $\mathbf{z}(1)=3.8$, we aim at computing an estimate for $E[\mathbf{x}(1) \mid \mathbf{x}(0), \mathbf{z}(1)]$, the expected value of $\mathbf{x}(1)$ given $\mathbf{x}(0)$ and $\mathbf{z}(1)$.

Theoretically, this problem could be solved by drawing a set of samples distributed according to the posterior p.d.f $p(\mathbf{x}(1) \mid \mathbf{x}(0), \mathbf{z}(1))$. However, in practice, it is a difficult task to drawn samples directly from the posterior p.d.f. The MonteCarlo method called Importance Sampling is a technique to approximate a target distribution $p(\mathbf{x})$ by using samples drawn from a importance distribution $q(\mathbf{x})$, similar ${ }^{4}$ to $p(\mathbf{x})$, by means of a process of weighting the samples [37]. According to the Importance Sampling it can be shown that an approximation for the posterior p.d.f $p(\mathbf{x}(1) \mid \mathbf{x}(0), \mathbf{z}(1))$ can be obtained by using a set of weighted particles, noted by $\left.\left\{\mathbf{x}^{(r)}(1), \omega^{(r)}\right\}\right|_{r=1} ^{N}$, drawn from an importance density $q(\mathbf{x}(1) \mid \mathbf{x}(0), \mathbf{z}(1))$ as follows:

$$
p(\mathbf{x}(1) \mid \mathbf{x}(0), \mathbf{z}(1)) \cong \sum_{i=1}^{N} \omega^{(r)} \delta\left(\mathbf{x}(1)-\mathbf{x}^{(r)}(1)\right)
$$

where $\delta(\tau)$ is the Dirac delta function and $\omega^{(r)}$ is the importance weight of sample $\mathbf{x}^{(r)}(1)$ and is given by:

$$
\omega^{(r)} \propto \frac{p\left(\mathbf{x}^{(r)}(1) \mid \mathbf{x}(0)\right) \cdot p\left(\mathbf{z}(1) \mid \mathbf{x}^{(r)}(1)\right)}{q\left(\mathbf{x}^{(r)}(1) \mid \mathbf{x}(0), \mathbf{z}(1)\right)} .
$$

Given a set of $N$ weighted particles $\left.\left\{\mathbf{x}^{(r)}(1), \omega^{(r)}\right\}\right|_{r=1} ^{N}$ an estimate for the expected value of $\mathbf{x}(1)$ is given by:

$$
E[\mathbf{x}(1) \mid \mathbf{x}(0), \mathbf{z}(1)] \approx \frac{\sum_{r=1}^{N} \mathbf{x}^{(r)}(1) \cdot \omega^{(r)}}{\sum_{r=1}^{N} \omega^{(r)}} .
$$

The most common choice for the importance density is the prior p.d.f $p(\mathbf{x}(1) \mid \mathbf{x}(0))$. However, since the prior p.d.f is independent of observations, the state space may not be efficiently explored. Indeed, for the problem considered in this section (see Problem 1) it can be observed that the probability of $\mathbf{x}(1) \in\left\{\mathbf{x} \in \mathbb{R}^{2}: x_{1}=3.3, x_{2} \leq 3.8\right\}$ is non-null. However, by using the prior p.d.f, the probability of obtaining a sample in the region $\left\{\mathbf{x} \in \mathbb{R}^{2}: x_{1}=3.3, x_{2} \leq 3.8\right\}$ is null. In order to overcome this drawback the following choice for the importance density is proposed:

$$
q(\mathbf{x}(1) \mid \mathbf{x}(0), \mathbf{z}(1))=\frac{T(\mathbf{x}(1) ; \mathbf{x}(0), \mathbf{z}(1)) \cdot p(\mathbf{x}(1) \mid \mathbf{x}(0))}{\Psi},
$$

where:

$$
\Psi=\int T(\mathbf{x}(1) ; \mathbf{x}(0), \mathbf{z}(1)) \cdot p(\mathbf{x}(1) \mid \mathbf{x}(0)) \partial \mathbf{x}(1),
$$

$T(\mathbf{x}(1) ; \mathbf{x}(0), \mathbf{z}(1))$ is a function used to incorporate informations given by $\mathbf{x}(0)$ and the observation $\mathbf{z}(1)$ to the importance density. These informations will be given by the support of the posterior p.d.f which can be computed by solving the conditional reachability problem [28]: given $\mathbf{x}(0)$ and $\mathbf{z}(1)$, compute the set of all $\mathbf{x}(1)$ that can be reached from $\mathbf{x}(0)$ through the transition model and that can lead to $\mathbf{z}(1)$ through the measurement function. This set, noted by $X_{1 \mid 1}$, represents the support of $p(\mathbf{x}(1) \mid \mathbf{x}(0), \mathbf{z}(1))$, mathematically:

$$
X_{1 \mid 1}=\mathcal{I}_{[\mathbf{A}]}\{\mathbf{x}(0)\} \cap \mathcal{I}_{[\mathbf{C}]}^{-1}\{\mathbf{z}(1)\} .
$$

From (29): $\mathcal{I}_{[\mathbf{A}]}\{\mathbf{x}(0)\}=\left\{\mathbf{x} \in \mathbb{R}^{2}: 1 \leq x_{1} \leq 4,2.5 \leq x_{2} \leq\right.$ $4\}$ and by using Algorithm 1, the inverse image of $\mathbf{z}(1)$ is

\footnotetext{
${ }^{4}$ A probability density $q(\mathbf{x})$ is similar to $p(\mathbf{x})$ if $\forall \mathbf{x}: p(\mathbf{x})>0 \Rightarrow$ $q(\mathbf{x})>0$
} 
given by: $\mathcal{I}_{[\mathbf{C}]}^{-1}\{\mathbf{z}(1)\}=\left\{\mathbf{x} \in \mathbb{R}^{2}: x_{1}=3.3, x_{2} \leq 3.8\right\} \cup$ $\left\{\mathbf{x} \in \mathbb{R}^{2}: x_{1}<3.3,2.8 \leq x_{2}^{\prime} \leq 3.8\right\}$. Thus, $X_{1 \mid 1}=R_{1} \cup R_{2}$, where:

$$
\begin{aligned}
& R_{1}=\left\{\mathbf{x} \in \mathbb{R}^{2}: x_{1}=3.3,2.5 \leq x_{2} \leq 3.8\right\}, \\
& R_{2}=\left\{\mathbf{x} \in \mathbb{R}^{2}: 1 \leq x_{1}<3.3,2.8 \leq x_{2} \leq 3.8\right\} .
\end{aligned}
$$

Given $X_{1 \mid 1}$ the function $T(\mathbf{x}(1) ; \mathbf{x}(0), \mathbf{z}(1))$ is defined as:

$$
T(\mathbf{x}(1) ; \mathbf{x}(0), \mathbf{z}(1))= \begin{cases}\delta\left(x_{1}-3.3\right), & \text { if } \mathbf{x}(1) \in R_{1}, \\ 1, & \text { if } \mathbf{x}(1) \in R_{2}, \\ 0, & \text { otherwise } .\end{cases}
$$

Remark 9: Note that, in order to have a non-null probability of generating a particle in region $R_{1}$ the function $T(\cdot)$ has an impulsive behavior in this region.

Now, let us compute the normalization constant $\Psi$. Note that (65) can be expressed as $\Psi=\psi_{1}+\psi_{2}$, where, (for more details on the following computations refer to Appendix A)

$$
\begin{aligned}
\psi_{1} & =\int_{R_{1}} T(\mathbf{x}(1) ; \mathbf{x}(0), \mathbf{z}(1)) \cdot p(\mathbf{x}(1) \mid \mathbf{x}(0)) \partial \mathbf{x} \\
& =\int_{R_{1}} \delta\left(x_{1}-3.3\right) \cdot p(\mathbf{x}(1) \mid \mathbf{x}(0)) \partial \mathbf{x} \\
& =p_{x_{1}}(3.3 \mid \mathbf{x}(0)) \cdot \int_{2.5^{-}}^{3.8} p_{x_{2}}\left(x_{2}(1) \mid \mathbf{x}(0)\right) d x_{2} \\
& =0.2250 \\
\psi_{2} & =\int_{R_{2}} T(\mathbf{x}(1) ; \mathbf{x}(0), \mathbf{z}(1)) \cdot p(\mathbf{x}(1) \mid \mathbf{x}(0)) \partial \mathbf{x} \\
& =\int_{1^{-}}^{3.3} p_{x_{1}}\left(x_{1}(1) \mid \mathbf{x}(0)\right) d x_{1} \cdot \int_{2^{-8^{-}}}^{3.8} p_{x_{2}}\left(x_{2}(1) \mid \mathbf{x}(0)\right) d x_{2} \\
& =0.4125
\end{aligned}
$$

where $p_{x_{1}}\left(x_{1}(1) \mid \mathbf{x}(0)\right)$ and $p_{x_{2}}\left(x_{2}(1) \mid \mathbf{x}(0)\right)$ are the marginal prior p.d.f of $x_{1}$ and $x_{2}$, respectively. Expressions for the marginal p.d.f are derived in Appendix A.

Remark 10: Note that, thanks to the hyperrectangles representation for the support of the posterior p.d.f $p(\mathbf{x}(1) \mid \mathbf{x}(0), \mathbf{z}(1))$, given by $R_{1}$ and $R_{2}$, it is possible to express a multiple integral as a product of single variables integrals, whose integrating functions are the marginal p.d.f $p_{x_{i}}\left(x_{i}(1) \mid \mathbf{x}(0)\right)$. By definition, the integral of a marginal p.d.f is a marginal cumulative density function (c.d.f). Expressions for the marginal c.d.f are derived in Appendix A.

If $\mathbf{x}(1)$ is sampled from the importance density (64) the probability of $\mathbf{x}(1) \in R_{1}$ is given by $P\left[\mathbf{x}(1) \in R_{1}\right]=$ $\psi_{1} /\left(\psi_{1}+\psi_{2}\right)$. Equivalently, $P\left[\mathbf{x}(1) \in R_{2}\right]=\psi_{2} /\left(\psi_{1}+\psi_{2}\right)$. Thus:

$$
P\left[\mathbf{x}(1) \in R_{1}\right]=0.3529, \quad P\left[\mathbf{x}(1) \in R_{2}\right]=0.6471 .
$$

The importance weight $\omega^{(r)}$ of a sample $\mathbf{x}^{(r)}(1)$ drawn from the importance density (64) can be computed by (78), in Appendix B. Algorithm 2 describes a procedure for generating a set of $N$ weighted particles representing the posterior p.d.f of the states of the system (59)-(60).

In order to test the efficiency of the approach, a simulation was carried out, where Algorithm 2 was used to generate sets of $N=10^{2}, 10^{3}$ and $10^{4}$ particles representing approximations for the posterior p.d.f of the system states. These sets

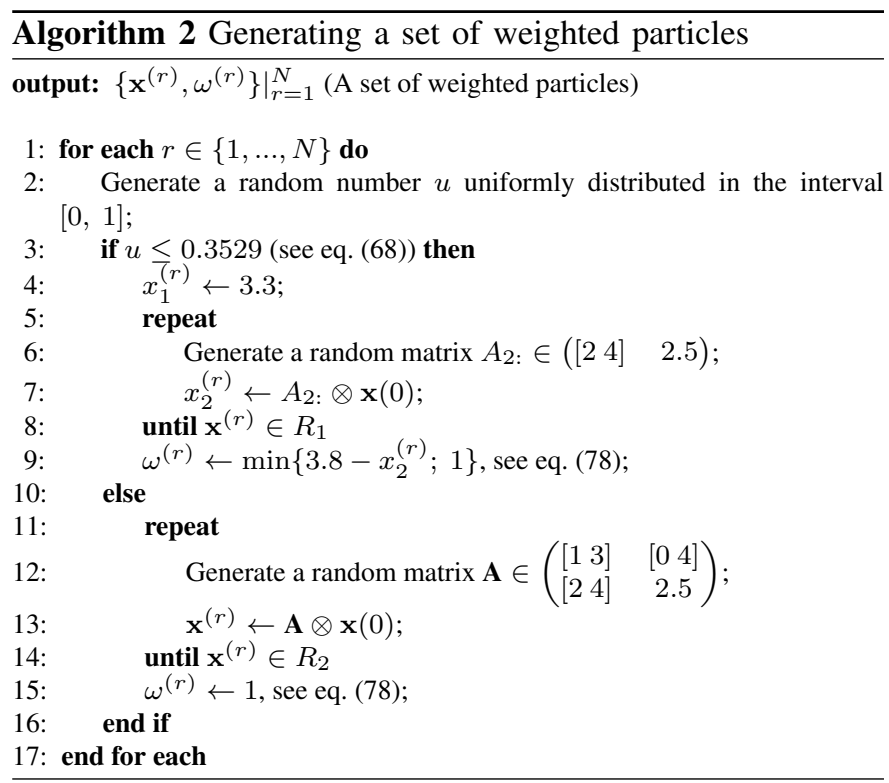

of particles were used to estimate the expected value of $\mathbf{x}(1)$ according to (63). The expected value was also analytically computed. The results can be observed in Table I.

TABLE I

EXPECTED VALUE OF $\mathbf{x}(1)$ (AVERAGE OVER 20 EXPERIMENTS)

\begin{tabular}{ccccc} 
State & \multicolumn{3}{c}{ Estimated Value } & Analytically \\
& $N=10^{2}$ & $N=10^{3}$ & $N=10^{4}$ & Computed Value \\
\hline$x_{1}$ & 2.5883 & 2.5865 & 2.5880 & 2.5873 \\
$x_{2}$ & 3.1530 & 3.1518 & 3.1524 & 3.1525 \\
\hline
\end{tabular}

\section{CONCLusions}

In this work we have presented an approach based on the interval matrices to compute the inverse image of a point w.r.t. an uMPL system. The algorithm presented has complexity $\mathcal{O}\left(n p^{n+1}\right)$, which is lower than the worst case complexity of the DBM approach to compute the inverse image of a point. Although the DBM approach is more general, in the sense that it can be applied to compute the inverse image of more general set than a single point, the approach presented in this work is particularly more interesting than the DBM approach in filtering problems. As future work the authors aim to generalize the approach presented in section IV in order to develop a recursive filtering procedure for MPL systems.

\section{APPENDIX A \\ Cumulative and Probability Density Functions of AN UMPL EQUATION}

Consider the uMPL equation given (25). In addition, consider that, the entries of matrix $\mathbf{A}$ are deterministic values or random variables uniformly distributed in the intervals given by $[\mathbf{A}]$. Thus probability density function (p.d.f) and 
the cumulative density function (c.d.f) of the non-deterministic entries, noted by $p_{i j}^{\mathbf{A}}(\tau)$ and $F_{i j}^{\mathbf{A}}(\tau)$, respectively, are given by:

$$
\begin{aligned}
& p_{i j}^{\mathbf{A}}(\tau)= \begin{cases}\left(\bar{a}_{i j}-\underline{a}_{i j}\right)^{-1} & \text { if } \underline{a}_{i j} \leq \tau \leq \bar{a}_{i j} \\
0 & \text { otherwise. }\end{cases} \\
& F_{i j}^{\mathbf{A}}(\tau)= \begin{cases}0 & \text { if } \tau<\underline{a}_{i j} \\
\frac{\tau-\underline{a}_{i j}}{\bar{a}_{i j}-\underline{a}_{i j}} & \text { if } \underline{a}_{i j} \leq \tau \leq \bar{a}_{i j} \\
1 & \tau>\bar{a}_{i j} .\end{cases}
\end{aligned}
$$

For the deterministic entries:

$$
p_{i j}^{\mathbf{A}}(\tau)=\delta\left(\tau-a_{i j}\right), \quad F_{i j}^{\mathbf{C}}(\tau)=u\left(\tau-a_{i j}\right),
$$

where $\delta(\tau)$ is the Dirac delta function and $u(\tau)$ is the step function. In [21, Appendix A] it is demonstrated that the cumulative density function of $\mathbf{z}(k)$ given $\mathbf{x}(k)$ is given by:

$$
F(\mathbf{z}(k) \mid \mathbf{x}(k))=\prod_{i=1}^{n} F_{z_{i}}(\mathbf{z}(k) \mid \mathbf{x}(k)),
$$

where $F_{z_{i}}(\mathbf{z}(k) \mid \mathbf{x}(k))$ is the marginal c.d.f of $z_{i}$, given by:

$$
F_{z_{i}}(\mathbf{z}(k) \mid \mathbf{x}(k))=\prod_{j=1}^{p} F_{i j}^{A}\left(z_{i}-x_{j}\right) .
$$

The p.d.f of $\mathbf{z}(k)$ is obtained by differentiating (72) successively with respect $z_{1}(k), \ldots, z_{n}(k)$, which yields:

$$
p(\mathbf{z}(k) \mid \mathbf{x}(k))=\prod_{i=1}^{n} p_{z_{i}}\left(z_{i}(k) \mid \mathbf{x}(k)\right),
$$

where $p_{z_{i}}\left(z_{i}(k) \mid \mathbf{x}(k)\right)$ is the marginal p.d.f of $z_{i}$, given by:

$$
p_{z_{i}}\left(z_{i}(k) \mid \mathbf{x}(k)\right)=\sum_{j=1}^{p} p_{i j}^{A}\left(z_{i}-x_{j}\right) \prod_{\substack{k=1, k \neq j}}^{p} F_{i k}^{A}\left(z_{i}-x_{k}\right) .
$$

\section{APPENDIX B}

\section{DERIVATION OF THE IMPORTANCE WEIGHTS EQUATION}

According to (62), if the importance density is given by (64), the importance weights are given by:

$$
\begin{aligned}
\omega^{(r)} & \propto \frac{p\left(\mathbf{x}^{(r)}(1) \mid \mathbf{x}(0)\right) \cdot p\left(\mathbf{z}(1) \mid \mathbf{x}^{(r)}(1)\right)}{T\left(\mathbf{x}^{(r)}(1) ; \mathbf{x}(0), \mathbf{z}(1)\right) \cdot p\left(\mathbf{x}^{(r)}(1) \mid \mathbf{x}(0)\right)} \cdot \Psi \\
& \propto \frac{p\left(\mathbf{z}(1) \mid \mathbf{x}^{(r)}(1)\right)}{T\left(\mathbf{x}^{(r)}(1) ; \mathbf{x}(0), \mathbf{z}(1)\right)} .
\end{aligned}
$$

According to (74), for the uMPL system (59)-(60):

$$
\begin{aligned}
p(\mathbf{z}(1) \mid \mathbf{x}(1))=p_{11}^{C}\left(z_{1}-x_{1}\right) \cdot F_{12}^{C}\left(z_{1}-x_{2}\right) & \\
& +p_{12}^{C}\left(z_{1}-x_{2}\right) \cdot F_{11}^{C}\left(z_{1}-x_{1}\right) .
\end{aligned}
$$

From (69) to (71), if $\mathbf{z}(1)=3.8$ then:

$$
\begin{gathered}
p_{11}^{\mathbf{C}}\left(z_{1}-x_{1}\right)=\delta\left(3.3-x_{1}\right), F_{11}^{\mathbf{C}}\left(z_{1}-x_{1}\right)=u\left(3.3-x_{1}\right), \\
p_{12}^{\mathbf{C}}\left(z_{1}-x_{2}\right)= \begin{cases}1 & \text { if } 2.8 \leq x_{2} \leq 3.8 \\
0 & \text { otherwise },\end{cases} \\
F_{12}^{\mathbf{C}}\left(z_{1}-x_{2}\right)= \begin{cases}0 & \text { if } x_{2}>3.8 \\
3.8-x_{2} & \text { if } 2.8 \leq x_{2} \leq 3.8 \\
1 & \text { if } x_{2}<2.8 .\end{cases}
\end{gathered}
$$

Hence, by substituting (67) and (77) in (76), the nonnormalized weights equation is given by:

$$
\omega^{(r)}= \begin{cases}\min \left\{3.8-x_{2}^{(r)} ; 1\right\}, & \text { if } \mathbf{x}^{(r)}(1) \in R_{1} \\ 1, & \text { if } \mathbf{x}^{(r)}(1) \in R_{2}\end{cases}
$$

\section{REFERENCES}

[1] C. Cassandras and S. Lafortune, Introduction to Discrete Event Systems, ser. SpringerLink Engineering. Springer US, 2009.

[2] F. Baccelli, G. Cohen, G. Olsder, and J. Quadrat, Synchronization and Linearity : An Algebra for Discrete Event Systems. Wiley and Sons, 1992.

[3] T. Brunsch, J. Raisch, and L. Hardouin, "Modeling and control of highthroughput screening systems," Control Engineering Practice, vol. 20:1, pp. 14-23, Jan 2012.

[4] B. Heidergott, G. Olsder, and J. van der Woude, Max Plus at Work: Modeling and Analysis of Synchronized Systems : a Course on MaxPlus Algebra and Its Applications, ser. Max Plus at work: modeling and analysis of synchronized systems : a course on Max-Plus algebra and its applications. Princeton University Press, 2006, no. v. 13.

[5] G. Cohen, S. Gaubert, and J.-P. Quadrat, "Max-plus algebra and system theory: Where we are and where to go now," Annual Reviews in Control, vol. 23 , pp. $207-219,1999$.

[6] M. Lhommeau, L. Hardouin, J.-L. Ferrier, and I. Ouerghi, "Interval Analysis in Dioid : Application to Robust Open Loop Control for Timed Event Graphs," in and, seville, dec 2005, pp. 7744-7749, doi:10.1109/CDC.2005.1583413.

[7] M. Lhommeau, L. Hardouin, and B. Cottenceau, "Optimal control for $(\max ,+)$-linear systems in the presence of disturbances," in Positive Systems, Proceedings of the First Multidisciplinary International Symposium on Positive Systems: Theory and Applications (POSTA 2003), Rome, Italy, Aug 2003, pp. 47-54.

[8] S. Amari, I. Demongodin, J. J. Loiseau, and C. Martinez, "Max-plus control design for temporal constraints meeting in timed event graphs," IEEE Transactions on Automatic Control, vol. 57, no. 2, pp. 462-467, Feb 2012.

[9] V. M. Gonçalves, C. A. Maia, and L. Hardouin, "On the steadystate control of timed event graphs with firing date constraints," IEEE Transactions on Automatic Control, vol. 61, no. 8, pp. 2187-2202, Aug 2016.

[10] R. D. Katz, "Max-plus (A,B)-invariant spaces and control of timed discrete-event systems," IEEE Transactions on Automatic Control, vol. 52, no. 2, pp. 229-241, Feb 2007.

[11] C. A. Maia, L. Hardouin, R. Santos-Mendes, and J. J. Loiseau, "A supereigenvector approach to control constrained max-plus linear systems," in 2011 50th IEEE Conference on Decision and Control and European Control Conference, Dec 2011, pp. 1136-1141.

[12] I. Necoara, B. De Schutter, T. van den Boom, and H. Hellendoorn, "Robust control of constrained max-plus-linear systems," Int. J. of Robust and Nonlinear Control, vol. 19, no. 2, pp. 218-242, Jan 2009.

[13] M. D. Loreto, S. Gaubert, R. D. Katz, and J. Loiseau, "Duality between invariant spaces for max-plus linear discrete event systems," SIAM J. on Control and Optimaztion, 2010.

[14] L. Hardouin, C. A. Maia, B. Cottenceau, and M. Lhommeau, "Observer design for $(\max ,+)$ linear systems," IEEE Trans. on Automatic Control, vol. 55 - 2, pp. $538-543,2010$.

[15] D. Luenberger, "An introduction to observers," IEEE Transactions on Automatic Control, vol. 16, no. 6, pp. 596-602, December 1971.

[16] M. DiLoreto, S. Gaubert, R. Katz, and J.-J. Loiseau, "Duality between invariant spaces for max-plus linear discrete event systems," SIAM Journal on Control and Optimization, vol. 48, no. 8, pp. 5606-5628, Dec. 2010.

[17] L. Hardouin, C. A. Maia, B. Cottenceau, and M. Lhommeau, "Maxplus Linear Observer: Application to manufacturing Systems," in , WODES'10, Sep. 2010, pp. 171-176.

[18] B. F. Heidergott, Max-Plus Linear Stochastic Systems and Perturbation Analysis (The International Series on Discrete Event Dynamic Systems). Secaucus, NJ, USA: Springer-Verlag New York, Inc., 2006.

[19] G. J. Olsder, J. A. C. Resing, R. E. D. Vries, M. S. Keane, and G. Hooghiemstra, "Discrete event systems with stochastic processing times," IEEE Transactions on Automatic Control, vol. 35, no. 3, pp. 299-302, Mar 1990.

[20] T. van den Boom and B. De Schutter, "Model predictive control for perturbed max-plus-linear systems," Systems \& Control Letters, vol. 45, no. 1, pp. 21-33, Jan 2002. 
[21] R. M. F. Cândido, R. Santos-Mendes, L. Hardouin, and C. Maia, "Particle filter for max-plus systems," European Control Conference, ECC 2013, 2013.

[22] R. M. F. Cândido and R. S. Mendes, "Filtro de partículas para sistemas max plus com densidade de importancia Ótima," in Anais do XX Congresso Brasileiro de Automática, 2014, pp. 3413-3420.

[23] D. F. Silva, R. S. Mendes, L. Hardouin, C. A. Maia, and B. Cottenceau, "Filtragem estocástica aplicada a sistemas max-plus lineares," in Anais do X Simpósio Brasileiro de Automaço Inteligente, 2011, pp. 1388-1393.

[24] Q. Lu, M. Madsen, M. Milata, S. Ravn, U. Fahrenberg, and K. G. Larsen, "Reachability analysis for timed automata using max-plus algebra," The Journal of Logic and Algebraic Programming, vol. 81, no. 3, pp. $298-$ $313,2012$.

[25] X. Allamigeon, S. Gaubert, and E. Goubault, "Inferring min and max invariants using max-plus polyhedra," in Proceedings of the 15th International Symposium on Static Analysis, ser. SAS'08, Berlin, Heidelberg, 2008, pp. 189-204.

[26] X. Allamigeon, U. Fahrenberg, S. Gaubert, R. D. Katz, and A. Legay, "Tropical fouriermotzkin elimination, with an application to real-time verification," International Journal of Algebra and Computation, vol. 24, no. 05, p. 569607, Aug 2014. [Online]. Available: http://dx.doi.org/10.1142/S0218196714500258

[27] D. Adzkiya, B. De Schutter, and A. Abate, "Computational techniques for reachability analysis of max-plus-linear systems," Automatica, vol. 53, no. 3, pp. 293-302, 2015.

[28] R. M. F. Cândido, L. Hardouin, M. Lhommeau, and R. S. Mendes, "Conditional reachability of uncertain max plus linear systems," Automatica, vol. 94, pp. 426 - 435, 2018. [Online]. Available: https://www.sciencedirect.com/science/article/pii/S0005109817305721

[29] K. Cechlárová, "Eigenvectors of interval matrices over maxplus algebra," Discrete Applied Mathematics, vol. 150, no. 1, pp. 2 - 15, 2005. [Online]. Available: http://www.sciencedirect.com/science/article/ pii/S0166218X0500096X

[30] K. Cechlárová and R. Cuninghame-Green, "Interval systems of max-separable linear equations," Linear Algebra and its Applications, vol. 340, no. 1, pp. 215 - 224, 2002. [Online]. Available: http://www.sciencedirect.com/science/article/pii/S0024379501004050

[31] H. Myšková, "Interval systems of max-separable linear equations," Linear Algebra and its Applications, vol. 403, pp. 263 - 272, 2005. [Online]. Available: http://www.sciencedirect.com/science/article/ pii/S0024379505000820

[32] R. M. F. Cândido, L. Hardouin, M. Lhommeau, and R. S. Mendes. (2019) An algorithm to compute the inverse image of a point with respect to a nondeterministic max plus linear system. [Online]. Available: http://perso-laris.univ-angers.fr/ renato/

[33] R. E. Moore and F. Bierbaum, Methods and Applications of Interval Analysis (SIAM Studies in Applied and Numerical Mathematics) (Siam Studies in Applied Mathematics, 2.). Soc for Industrial \& Applied Math, 1979.

[34] T. Brunsch, L. Hardouin, C. A. Maia, and J. Raisch, "Duality and interval analysis over idempotent semirings," Linear Algebra and its Applications, vol. 437, pp. 2436-2454, 2012.

[35] L. Hardouin, B. Cottenceau, M. Lhommeau, and E. Le Corronc, "Interval systems over idempotent semiring," Linear Algebra and its Applications, vol. 431, no. 5-7, pp. 855-862, Aug. 2009, doi:10.1016/j. LAA.2009.03.039.

[36] G. L. Litvinov and A. N. Sobolevskiī, "Idempotent interval analysis and optimization problems," Reliable Computing, vol. 7, no. 5, pp. 353-377, 2001.

[37] B. Ristic, S. Arulampalam, and N. Gordon, Beyond the Kalman Filter. Artech House, 2004.

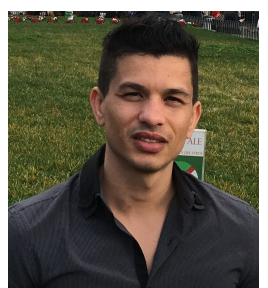

Renato Markele Ferreira Cândido received the B.S. degree in electrical engineering from the Universidade Federal de Viosa (UFV, Brazil, 2011), the M.S degree from Universidade Estadual de Campinas (UNICAMP, Brazil, 2013) and the Ph.D. degrees from Universidade Estadual de Campinas (UNICAMP, Brazil, 2017), as well as from the University of Angers, France, in 2017. His current research interests include Discrete Event Dynamic Systems, Dioid Algebra, Stochastic Filtering and Reachability Analysis.

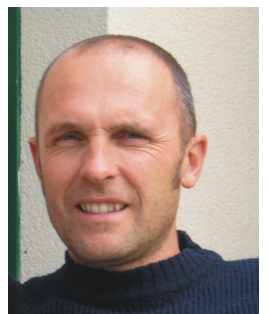

Laurent Hardouin received the degrees of Master and Ph.D. in Acoustic and Control from the University of Poitiers, France, in 1990 and 1993, respectively. He received the Habilitation à Diriger des Recherches from the University of Angers, France, in 2004. He is currently a Full Professor of Polytech Angers at the University of Angers, France. He specializes in discrete event systems, max-plus algebra, and interval analysis with applications to computer networks, manufacturing systems, transportation systems, and robotics.

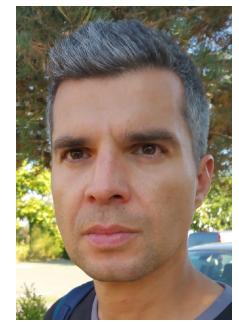

Mehdi Lhommeau was born in 1975 . He received the Ph.D. degree from the University of Angers, France, in 2003. He is currently an Associate Professor at the University of Angers. His main research interests include modeling and control of timed discrete event systems in max-plus algebra with applications in manufacturing systems and computer networks.

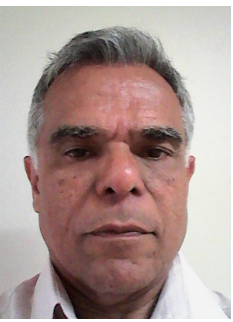

Rafael Santos Mendes received the Electrical Engineering degree from the State University of Campinas, Brazil (UNICAMP, 1981), the M.S. degree from the State University of Campinas (UNICAMP, 1984) and the Docteur de lUniversité degree from the Paul Sabatier University/LAAS-CNRS (Toulouse, France, 1988). Currently, he is an Associate Professor in the School of Electrical and Computer Engineering, UNICAMP. His research interests include Discrete Event Dynamic Systems, Dioid Algebra, Stochastic Filtering and Modal Logic. 